\title{
Studies of Transport Properties of Fractures Final Report
}

June 30, 2006

U.S. Department of Energy Award No: DE-FG02-98ER14906

Principal Investigator: Stephen R. Brown, New England Research, 331 Olcott Driver, Ste L1, White River Junction, Vermont 05001.

Collaborating Principal Investigator: Harlan W. Stockman, Sandia National Laboratories, Albuquerque, New Mexico.

Other Senior Collaborators: Ronald Bruhn, University of Utah, Salt Lake City, Utah; Arvind Caprihan, New Mexico Resonance, Albuquerque, New Mexico; Carlos Jove-Colon, Sandia National Laboratories, Albuquerque, New Mexico;

Participating Researchers: Katherine Ebel, Joel Johnson, Anjana Khatwa, Todd Myse, Carl Renshaw.

Purpose: To study the relationships between the fluid flow and transport properties of fractured rock and the topography of the fracture surfaces.

Individual Projects Funded Under This Grant:

- The Role of Fracture Intersections in the Flow and Transport Properties of Rock. Funded in the years 1999-2002.

- Evolution ofFracture Permeability. Funded in the years 2002-2006.

Disclaimer: Any opinions, findings, and conclusions or recommendations expressed in this document are those of the author and do not necessarily reflect the views of the Department of Energy.

This paper was written with support of the U.S. Department of Energy under Contract No. DE-FG0298ER14906. The Government reserves for itself and others acting on its behalf a royalty-free, nonexclusive, irrevocable, worldwide license for Governmental purposes to publish, distribute, translate, duplicate, exhibit and perform this copyrighted paper. 


\section{Contents}

1 Executive Summary $\quad 3$

2 Introduction $\quad 5$

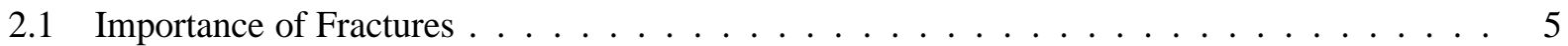

2.2 Channeling in Single Fractures $\ldots \ldots \ldots \ldots \ldots$

2.3 Solute Mixing Rules at Fracture Intersections $\ldots \ldots \ldots \ldots$

2.4 Channeling in Fracture Networks . . . . . . . . . . . . . . . . . . . . 9

2.5 Geochemical Evolution of Fracture Permeability $\ldots \ldots \ldots \ldots$

3 Proposed Research $\quad 12$

4 Relationship to DOE/OBES mission $\quad 12$

5 Techniques Used and Resources Applied 14

6 Summary of Results $\quad 14$

6.1 Mixing in Natural Fracture Intersections . . . . . . . . . . . . . . . . . . . . . 14

6.2 Mixing in Synthetic Fracture Intersections . . . . . . . . . . . . . . . . . 16

6.3 Mixing in Fracture Networks . . . . . . . . . . . . . . . . . . . . 21

6.4 MRI Studies of Mixing Within Intersections (Collaborative Project) . . . . . . . . . . . 24

6.5 Formation of Fracture Networks (Collaborative Project) . . . . . . . . . . . . . . . 26

6.6 Field Measurement of Fracture Aperture . . . . . . . . . . . . . . . . . . . . 27

6.7 Dissolution in Fractures . . . . . . . . . . . . . . . . . . . . . 28

6.8 Observations in Vein Systems (Collaborative Project) . . . . . . . . . . . . . . . 33

6.9 Precipitation in Fractures (Future Directions) $\ldots \ldots \ldots \ldots$

7 Proposed Future Work $\quad 41$

8 Publications Resulting From This Project 42

9 Commercial Products Developed $\quad 44$

10 Principal Project Personnel 45

$\begin{array}{ll}\text { References } & 47\end{array}$ 


\section{Executive Summary}

Objectives: Fluid flow in fractured rock is an important phenomenon to understand in connection with oil, gas, and geothermal energy production and protection of groundwater supplies through safe disposal and containment of toxic wastes. Both discrete fracture and effective continuum approaches to the modeling of flow and transport in fracture networks need to be based on a sound understanding of flow in single fractures, how flow is transferred across intersections, and how multiple fractures form a network. We suggest that due to surface roughness and the consequent channeling in single fractures, volume flow and solute mixing behavior at fracture intersections will be considerably different from that expected based on simple assumptions and experiments with smooth-walled fractures.

Therefore, the first objective of this project is to gain a more complete understanding of flow channeling by physically modeling and analyzing several configurations of flow and transport through fracture intersections.

The nature of fluid/rock chemical interaction within a fracture system is fundamental to determining the creation or loss of permeability. We suggest that, due to surface roughness and the resulting fluid flow channeling in single fractures and in fracture intersections, the fracture system permeability will decrease or increase due to chemical precipitation or dissolution. Due to flow channeling, the fluids or the solutes they contain (which can interact chemically with the wall rock) are in contact with smaller areas of the fracture surfaces and for less time than for uniform flow.

Therefore, the second objective of this study is to determine how chemical interaction between the pore fluid and the rock controls the evolution of fracture system permeability.

Project Description: We address several problems concerning flow and transport through fracture intersections using a combination of numerical modeling, quantitative measurements, and the visual observation of processes in real rough-walled fractures. Our observations include: channeling in single fractures, channeling within and through single fracture intersections, and channeling through fracture networks. We have also advanced our understanding of changes in fracture permeability through time and develop a predictive capability by constructing physical models and analyzing several configurations of flow and transport of chemically active fluids through channels in single fractures and through fracture intersections.

This represents a joint project among staff at New England Research and Sandia National Laboratories and has utilizes quantitative observations of flow channels within rough-walled fractures (evolving through dissolution and precipitation), numerical modeling, and quantitative observations from fractures and veins in drill core.

This work made use of our abilities to (1) construct high quality replicas of actual fractured rock in transparent plastics, (2) simulate surface roughness with the computer, (3) construct bench-scale fracture systems, (4) observe actual fracture apertures, fluid flow, and solute transport with quantitative visualization techniques, and (5) conduct detailed numerical simulations of flow and transport in heterogeneous media. For this work we employ a combination of quantitative measurements, digital video imaging and magnetic resonance imaging (MRI) observations of flow through real rough-walled fractures, and numerical modeling via lattice Boltzmann (LB) methods.

Results: Several natural fracture intersections are replicated in transparent epoxy, while synthetic intersections are made from textured glass. Laboratory experiments of single-phase mixing of solutes are performed by flowing water and dye solutions through the specimens. We find that in intersecting natural fractures the degree of average mixing is greater than predicted by parallel plate streamline routing. Apertures at the intersection vary spatially, resulting in highly variable flow rates around the intersection. This leads to multiple pure fingers of each solution both bending around and crossing straight through an intersection. While the 
outlet fluids are heterogeneous (not uniformly mixed), the average degree of mixing is higher than expected for hydraulically equivalent parallel plates.

In this study we compare the experimental flow and mixing results to numerical methods using the experimentally-observed intersection geometry. The Reynolds equation for 2-dimensional flow is solved simultaneously for flow in both variable aperture fractures, with source and sink terms creating an intersection between the two. Numerical simulations for the textured glass laboratory specimen agree well with the laboratory experiments, both in the amount of average mixing and in the spatial distribution of dye streamlines.

We also consider the effect of surface roughness on solute dispersion within large networks of fractures. A numerical model of a fracture network is created by taking the detailed properties and behaviors observed for the textured glass sample and replicating it many times to form a regular network or lattice. Simulations of flow through this network result in dispersion which is intermediate between complete mixing and pure streamline routing. We find that identical network-wide dispersions can be obtained if we either (1) consider all details of flow and mixing in the rough fractures or (2) we simply assume that all components in a network of identical parallel-plate fractures each have the same effective mixing ratio as the rough-walled ones.

Using MRI we attempt to obtain structural information, measure flow velocities, and quantify mixing in three dimensions. Beginning with a parallel plate specimen we develop techniques which we then applied to experiments on intersecting natural rock fractures. We find that it is possible to make accurate aperture measurements when there is no flow and to make adequate flow velocity measurements away from the intersection. However, with the available techniques and equipment, we can not image flows near to or within the intersection due to signal loss effects in small apertures typical of these natural specimens especially when the liquid is flowing. Likewise, measurements of mixing processes are only possible in the parallel plate fracture specimen. Useful data can be gained in all cases, however, if we sacrifice spatial resolution and only measure properties in a statistical sense.

For fracture dissolution studies, our experimental model consists of plaster samples pressed with a constant force against an inert textured fracture surface. Pore fluids ranging from unsaturated to supersaturated are introduced to one end of the sample in order to actively alter the fracture surface topography. We analyze features on the evolving fracture surfaces including high-flow dissolution channels, dissolution-resistant plateaus, and caverns formed from precipitate. We use finite difference and lattice Boltzmann (LB) methods to calculate pore fluid flow direction and magnitude over the entire sample surface.

The flow channel networks evolve from a homogeneous system to one more (self) organized and complex. LB methods predict the formation of major dissolution features seen in the experiments, such as long undissolved "stringers" in the lee of obstacles.

LB predicts that dendrites may grow along fracture surfaces under saturated conditions. This form of growth can be the major cause of decreasing permeability, even when the added solid fraction is small. LB predicts dendrites will grow toward time-varying regions of fast flow. Based on this idea, some larger-scale numerical models of permeability evolution in actively dissolving or infilling fracture are being developed. The models studied so far show a self-organization of flow channel structure and nearly linear changes in fluid permeability with time.

We substantiate these findings by obtaining quantitative observations from fractures and veins in drill core obtained from Awibengkok, Indonesia. Images of veins are analyzed for geometrical properties including variations in vein width, the roughness of vein walls, and the minimum aperture distribution. Most veins developed by episodic fracturing, fluid flow, and mineralization, implying that void space and permeability varied markedly over the life of the vein. Using our observations from the veins split core and thin sections we present a series of four qualitative permeability models which differ depending upon the history of fracturing and mineralization.

Through the course of this project the initial goals are met by identifying and quantifying all key processes. New directions of research are identified, leading to a plan for future work. 


\section{Introduction}

In this document we review our studies of the relationships between the fluid flow and transport properties of fractured rock and the topography of fracture surfaces. During this project we have endeavored to physically model and analyze several configurations of flow and transport of inert and chemically active fluids through channels in single fractures and through fracture intersections. This was an integrated program utilizing quantitative observations of fractures and veins in drill core, quantitative and visual observations of flow and chemical dissolution and precipitation within replicas of real rough-walled fractures and fracture intersections, and numerical modeling. This work represents a collaborative project between S.R. Brown of New England Research (NER) and H.W. Stockman of Sandia National Laboratories (SNL). The primary division of labor (although with considerable overlap) was that the experimental and field studies were performed at NER and the lattice Boltzmann (LB) numerical studies were performed at SNL. This report primarily emphasizes NER's contribution with only some mention of pertinent LB modeling results.

\subsection{Importance of Fractures}

Fluid flow in fractured rocks is a subject of primary importance to hazardous waste isolation, environmental remediation, oil and gas production from fractured reservoirs, reservoir stimulation by hydrofracturing, geothermal energy extraction, and the formation of vein fillings and ore deposits [e.g., Nelson, 1985; Burns, 1990; USGS Red-Book Conference, 1993; Brown and Bruhn, 1996; Committee on Fracture Characterization and Fluid Flow, 1996]. For these reasons, considerable effort has been placed on the characterization and modeling of flow in fractures and fracture systems.

Many studies have shown that fluid flow and solute transport occur mainly through channels within individual fractures. Additionally, select fractures of a network may take most of the flow, thus forming a larger-scale channel structure. Within this multi-level system of channels, fluid can travel much faster than might otherwise be expected from the mean velocity or uniform flow in idealized constant-width fractures. In our previous work, we have found that fluid velocities vary among different channels by many orders of magnitude and that the maximum velocity far exceeds the mean [Brown et al., 1998]. As flow encounters intersections between fractures the continuity of flow will be disrupted and the intersections may act as flow channels themselves. Due to natural surface roughness, mixing of solutes within single fractures, fracture intersections, and fracture networks is a complex process and deviates significantly from predictions of idealized intersecting parallel plate fractures.

Fracture flow and accompanying solute transport in volcanic rocks have been identified as major sources of uncertainty for performance assessment at Yucca Mountain, Idaho National Engineering and Environmental Laboratory, and the Hanford Site. Fracture flow paths in shales are an important source of uncertainty for contaminant transport at Oak Ridge National Laboratory. We lack a fundamental understanding of the processes controlling transport of multiple immiscible phases in fractures, such as the combination of water, air, and non-aqueous phase liquid pollutants (LNAPL's or DNAPL's). These deficiencies hinder site characterization and pollution remediation activities at many DOE sites, or make it difficult to justify regulatory decisions that rely on natural attenuation to mitigate public exposure.

Oil, gas, and geothermal energy extraction endeavors in fractured rock primarily rely on the fractures as conduits for the transport of fluids from the extremities of the reservoir to the borehole [Committee on Fracture Characterization and Fluid Flow, 1996]. The matrix often stores the hydrocarbons, thermal energy, or disseminated ore and thus the fractures are relied upon for their high permeability. One objective of energy exploration in fractured rocks is to find areas of intense fracturing that are capable of high flow and efficient transport to the extraction wells. The interconnections of fractures are extremely important to determining the accessibility of a well to the reservoir. Channeling of flow through fractures, fractures intersections and fractures networks plays an important role in the economics of this process. Furthermore 
scaling (the buildup of precipitants) can greatly reduce the permeability of the fractured host rocks, as well as the mechanical extraction system, as hot water from one realm is forced through the apertures of a realm that is grossly out of chemical equilibrium.

Here we summarize some of the key components and issues regarding flow channeling in fractured rock.

\subsection{Channeling in Single Fractures}

Fluid flow and transport in rock fractures have been commonly described by the parallel plate model, where the fracture surfaces are smooth with a constant separation or aperture. For this geometry, the steady state solution of the Navier-Stokes equations for laminar flow yields the cubic law, where the volume flow rate is proportional to the cube of the aperture [e.g., Iwai, 1976].

The parallel plate model can only approximate flow through real fractures. Real fracture surfaces are not smooth parallel plates but are rough and contact each other at discrete points [e.g., Brown and Scholz, 1985; Brown, 1995]. Fluids and the solutes they contain will take tortuous paths when moving through a real fracture; thus deviations from predictions of the parallel plate model are expected. Taking the spatial variation of the aperture into consideration, laminar flow between rough surfaces has been studied theoretically, numerically, and experimentally [e.g., Iwai, 1976; Gangi, 1978; Kranz et al., 1979; Brace, 1980; Tsang and Witherspoon, 1981; Walsh, 1981; Brown, 1987; Pyrak-Nolte et al., 1987, 1988; Brown, 1989; Stesky, 1986; Zimmerman et al., 1992; Olsson and Brown, 1993; Brown et al., 1995; Walsh et al., 1997]. These studies confirm that surface roughness plays an important role and can lead to significant departures from the parallel plate model. One of the more interesting processes identified is the restriction of flow to a system of narrow channels within the fracture plane.

A field experiment in the Stripa mine, Sweden [Bourke, 1987], demonstrated that flow in a single fracture took place mainly in a few widely separated channels. Five parallel holes were drilled in the fracture plane along the interface between the two contacting fracture surfaces. Each of these holes was pressurized in turn. During these pressurization episodes, various packed-off intervals were monitored for flow. Over a total fracture area of approximately $2 \times 3$ meters, two large channels comprising about $10 \%$ of the sample area and separated by approximately 1 meter were responsible for most of the flow.

Pyrak-Nolte et al. [1987] observed smaller-scale channels and tortuosity by injecting molten Wood's metal into a single fracture, and then opening the fracture when the metal had solidified. Brown and Scholz [1985] and Brown [1995] studied the topography of many natural rock joint surfaces and computed the apertures. With the aid of these data, a simple mathematical model of rough surfaces was derived. This model was used to create realistic simulated apertures. Solutions of fluid flow equations through these simulated fractures show distinct geometry-controlled channeling [Brown, 1987, 1989; Thompson and Brown, 1991].

We have directly observed flow and transport channels in actual fractures [Brown et al., 1998]. Digitized optical images were used to observe wetting, saturated flow, and drying of a fracture specimen (Figure 1). These video imaging techniques showed distinct and strong channeling of the flow at the sub-millimeter to several-centimeter scale. In this same study, pulsed magnetic resonance imaging (MRI) was used to observe the flow in the same fracture specimen. The probability distribution of the measured velocities is broad, somewhat bimodal, and ranges over many orders of magnitude. The mean velocity within this region was found to be $3.2 \mathrm{~cm} / \mathrm{s}$ and the maximum velocity was $18.3 \mathrm{~cm} / \mathrm{s}$. Fluid velocities measured simultaneously at various locations in the fracture plane during steady-state flow range over several orders of magnitude, with the maximum velocity a factor of 5 higher than the mean velocity.

\subsection{Solute Mixing Rules at Fracture Intersections}

A component of fundamental importance for accurate modeling of flow and transport in fracture networks is the definition of the rules for the inflow, mixing, and outflow of solutes at the fracture intersections. 


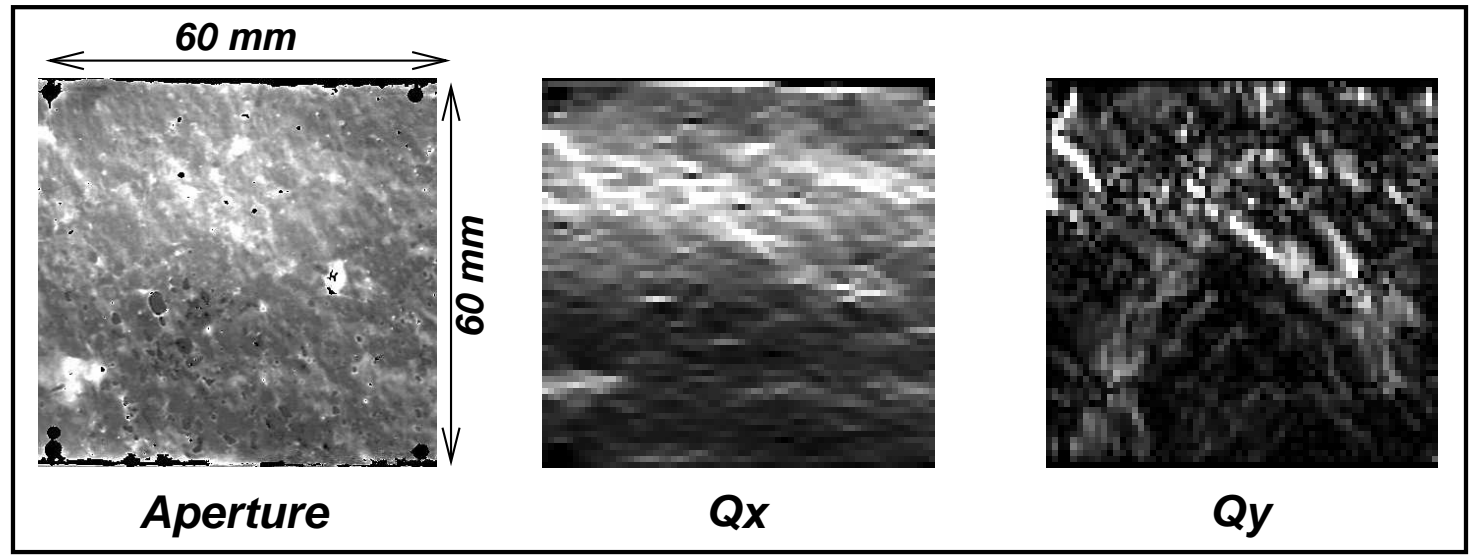

Aperture and Flow Rate Geometries

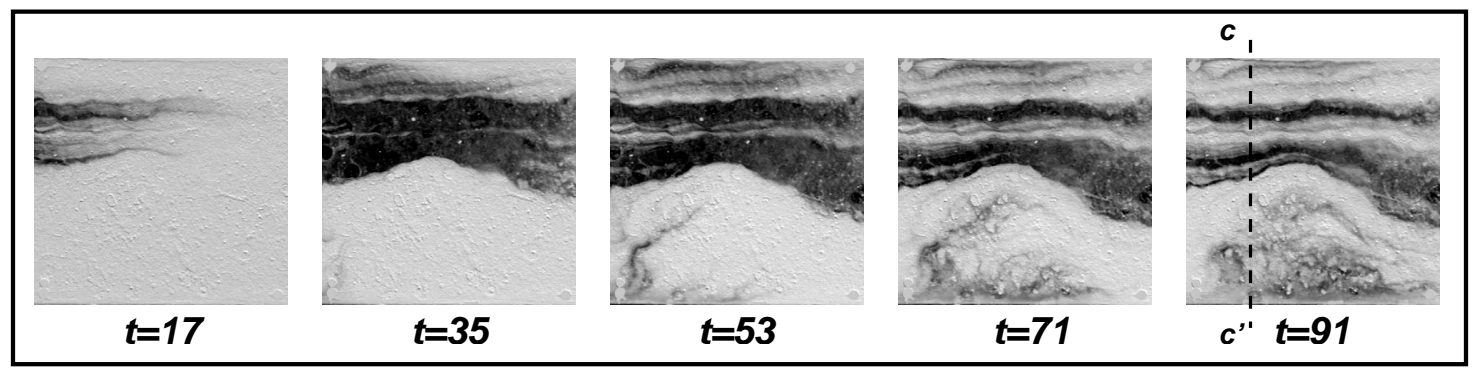

Time Sequence of Dye Flow

Figure 1: An example of direct observations of flow channeling in single fractures [Brown et al., 1998]. Top: A flow cell was constructed from a replica of a natural fracture in transparent epoxy resin. The Lambert-Beer law was used to derive the fracture aperture. This aperture distribution was used in a finitedifference Reynolds equation simulator to calculate the $\mathrm{x}$ and $\mathrm{y}$ components of flow velocity. Flow channels of various scales are apparent, with a dominant high flow channel appearing from left to right across the top of the images. Quantitative flow velocity measurements using pulsed MRI in this specimen showed nearly identical patterns with several orders of magnitude variations in velocities. Bottom: The flow cell was used to observe dye flow at various times through the fracture. Dye flow patterns are heterogeneous and mimic the flow velocity distribution patterns, with distinct and strong channeling at the sub-millimeter to several-centimeter scale.

These mixing rules must be assumed for numerical modeling to proceed. There are four principal types of fracture junctions considered: one incoming, one outgoing, continuous, and discontinuous (Figure 2). As fluid flows into a fracture intersection and is distributed to the outlet paths, the redistribution of solute carried by the fluid is determined by the mixing behavior at the intersection. Three distinct mixing behaviors are considered: complete mixing, streamline routing, and forced mixing (Figure 3). Most fracture network models assume either complete mixing [Schwartz et al., 1983; Travis, 1984; Huang and Evans, 1985] or advection-controlled streamline routing [Wilson and Witherspoon, 1976; Hull and Koslow, 1986; Robinson and Gale, 1990]. The mixing rule chosen determines the final distribution of solute in large-scale simulations of fracture systems [e.g. Endo et al., 1984; Hull et al., 1987; Robinson and Gale, 1990].

The question of which mixing rule should be applied and under which circumstances has been reviewed and considered in detail by $L i$ [1995] for the case of simple intersections of smooth parallel-plate fractures. The mixing rule is a relationship between the mixing ratio and the flow and diffusion processes operating 


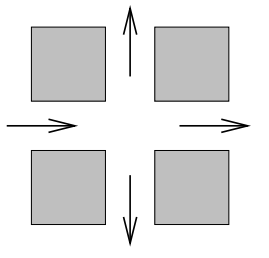

(a) one incoming

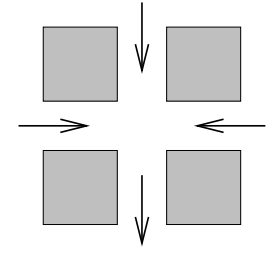

(b) one outgoing

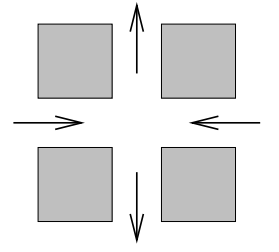

(c) discontinuous

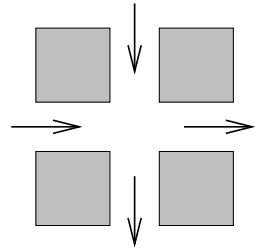

(d) continuous

Figure 2: Flow types in fracture intersections (after Küpper et al. [1995]).

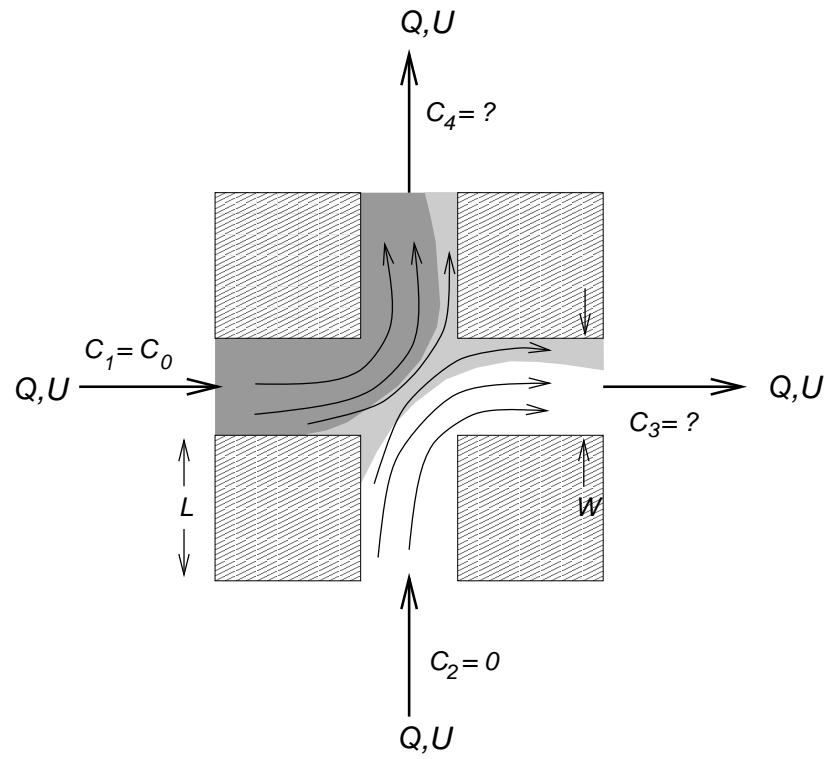

Figure 3: Idealized mixing behaviors at a continuous fracture intersection. The aperture of the fractures is $W$. Flow enters and leaves each fracture with volume flow rate $Q$ and average velocity $U$. Fluid with "black" solute at concentration $C_{0}$ enters from fracture 1, and "white" fluid with no solute enters from fracture 2. In general there will be a combination of pure transport along streamlines (black remains black and white remains white) and mixing by diffusion (gray fluid in center). Pure streamline routing is the case where $C_{3}=C_{0}$ and $C_{4}=0$. Complete mixing is where $C_{3}=C_{4}=0.5 C_{0}$. Not shown is the case of forced mixing where a partition is placed in the intersection to always direct some flow straight through the intersection, say from fracture 1 to fracture 3 .

in the fracture intersection. For the case of a continuous intersection, Stockman et al. [1997b] define the mixing ratio as (see Figure 3):

$$
M_{r}=\frac{C_{3}}{C_{1}} \approx \frac{C_{3}}{C_{3}+C_{4}} .
$$

Based on experimental evidence, $L i$ [1995] finds that, for a continuous intersection with equal flow rates in both fractures, the mixing ratio depends on Peclet Number, $P_{e}$, defined as:

$$
P_{e}=\frac{(\sqrt{2} / 2) W U}{D}
$$

where $(\sqrt{2} / 2) W$ is the half diagonal length of the junction, $U$ is the fluid velocity, and $D$ is the coefficient for molecular diffusion of the solute. The Peclet Number indicates the relative importance of advective to 


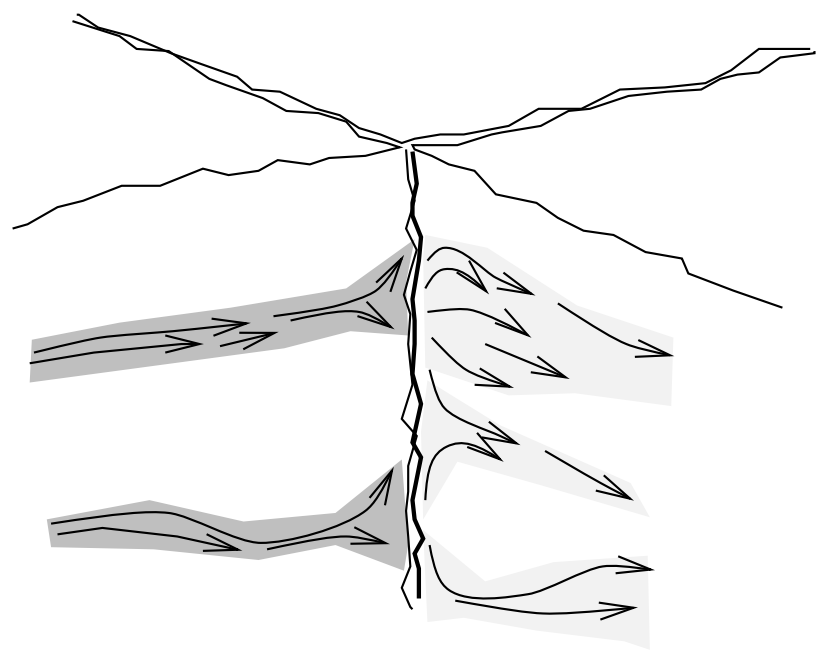

Figure 4: Conceptual view of channeling at a fracture intersection. Dominant channels in the inlet fracture do not match those in the outlet fracture, leading to complex mixing and channeling behavior along the intersection. We expect all mixing process (streamline routing, diffusion-controlled mixing, and forced mixing) to operate in the fracture planes as well as in and along the intersections.

diffusive processes for moving solutes. $L i$ [1995] proposes a mixing model, that quantitatively relates the mixing ratio for a simple fracture intersection to the Peclet number.

While illustrative, the previous studies on mixing behavior considered only the idealized 2-D geometry of two straight-walled, smooth fractures with constant aperture intersecting at high angles. The channeling behavior of real fracture intersections will be much more complex than the idealized parallel plate geometry (Figure 4). Channeling of flow within each rough fracture, where the principal aperture channels may not align, or the potential flow along the fracture junction itself are important processes to be considered in some detail. As with these previous studies concerning the transport and mixing of solutes, we expect the motion and redistribution of multiple immiscible phases (e.g. DNAPL's and LNAPL's) through fracture intersections to be strongly influenced by the geometry of the fracture intersections.

\subsection{Channeling in Fracture Networks}

Based on a review of theoretical and experimental studies of channeling in single fractures, Tsang and Tsang [1987] and Tsang et al. [1988] presented a conceptual model of channel flow through fractured media. They envisioned a system of one-dimensional channels interconnecting through the various fractures in the network. It is assumed that each channel has a variable aperture along its length and that the mean aperture is different from one channel to the next. They suggested that laboratory measurements of dispersion from tracer breakthrough curves can identify the geometric characteristics of the more conductive channels (mean and variance of aperture). Since flow in a channel system is highly heterogeneous, they identified the need in both field and laboratory experiments to monitor the transport in the fracture plane along lines perpendicular to flow rather than at a few selected points. These sampling lines should be long enough to cover several correlation lengths of the channel system.

Field evidence indicates that solute transport within fractured rock occurs in a limited subset of the fractures comprising a network, thus forming another type of channel system [Cacas et al., 1990; Abelin et al., 1991]. These preferred paths reflect, to some degree, the arrangement of higher permeability connections within the network [Parney and Smith, 1995]. Abelin et al. [1991] have observed that water flow can be highly uneven throughout fractured rock masses, with large dry areas extending over many tens of meters. 
To understand this effect, Sanderson and Zhang [1999] performed simulations of elastic deformation of fracture networks under differential stress and increasing pore fluid pressure. They showed how certain critically-located fractures in the initial (apparently random) network dilate to form potential high flow rate channels.

Therefore, due to channeling through fracture networks, field-scale flow simulation models and analyses of field data that rely on porous medium approximations will likely give unknown and potentially large errors for both flow and transport.

\subsection{Geochemical Evolution of Fracture Permeability}

Up to this point we have demonstrated that fluid flow and transport in fractures is characterized by heterogeneity at a variety of scales. For a single fluid phase, irregular aperture geometry gives rise to channelized flow in single fractures, with a wide range of velocities present. A mixture of multiple immiscible phases, exacerbates this effect. When the fractures intersect, with each containing distinct solutions (solutes) that are miscible, the interacting channels create complex mixing behaviors, such that when diffusion is minimal the "mixed" fluids exiting the intersection are actually predominantly intercalated streamers of the two pure inlet fluids. These ribbons of solute are then moving with the wide spectrum of velocities characteristic of rough-aperture fracture flow. Furthermore, if one takes a step back and looks at the network of fractures, a larger-scale channel structure becomes apparent due to irregularities in the distribution of the fracture apertures throughout the network. Thus the entire system has a juxtaposition of fluid volumes, velocities, and compositions.

If the fluids are chemically active, then fracture system channel structure and thus the average permeability cannot be treated as a static property. For many applications, we must then consider how fracture permeability evolves through time as chemical interaction occurs between the pore fluid and the fracture walls. When fluid/rock chemical interaction results in either dissolution or precipitation of minerals, the geometry of the pore and permeability system will change. Aside from considerations of heterogeneous pore fluid chemistry, the heterogeneity in the fluid flow rate and the geometry of the flow channels will determine the evolution of the open fluid pathways. For example, we assume that veins represent once permeable fractures which have since been filled with mineral precipitates. The mechanisms leading to the ultimate permeability reduction may depend on both precipitation and dissolution.

Consideration of such geochemical evolution of fracture permeability has both important economic and environmental consequences.

In-situ solution mining - leaching of ore bodies Solution mining or in-situ leaching is a technique for extracting minerals directly from an ore body without blasting or excavation. An in-situ mine consists of a series of injection and recovery wells through which a weak acid or base solution (depending on the particular situation) is circulated to dissolve the mineral of interest in the subsurface. The mineral components are then recovered in a surface processing plant by a technique such as electrowinning. Solution mining is generally most suitable for small or low grade ore deposits, particularly uranium and copper, which would otherwise not be economical to mine using conventional open cut or underground techniques.

There are many production and environmental safety issues associated with these operations, for which knowledge of dissolution and precipitation processes in both matrix and fractures is required. For example, Harpalani [2000] studied rock samples both before and after several cycles of leaching at an in-situ copper mining operation. Results showed that permeability and copper production first increased, stabilized for a time, then significantly decreased through about eight months of operation. This production history was found to correspond to an initial increase in the number of fractures and the typical fracture apertures, then eventual clogging of the fractures due to precipitation of sulfates and production and motion of fine particles. 
Origin of ore deposits The origin of ore deposits involves both dissolution and precipitation issues in the presence of moving aqueous fluids. Guilbert and Park [1986] divide the question of how ore deposits are formed into 4 separate topics: (1) source and character of the fluids themselves, (2) sources of primary ore constituents and how they make it into solution, (3) migration of ore-bearing fluids, and (4) deposition of ore minerals from solution.

The mining and economic geology literature has many examples of the occurrence of ore deposits and other mineralization in and around fracture and fault intersections [e.g., Knopf, 1929; Eckel, 1949; Peters, 1993]. Mine maps commonly show duplex structures, where two or more faults link together or intersect. The quartz-gold veins of the Mother Lode are a case in point - the ore was concentrated in many cases along fault intersections rather than in voids between fault walls [Knopf, 1929]. Clearly, not only the geometry of the intersections, but the fluid flow pathways through them are important in determining the location, distribution, and grade of ores.

Fault-vein systems are typically composed of numerous individual veins and whisker-like veinlets that form where mineral-rich fluids move along larger fractures and infiltrate brecciated rock [Knopf, 1929; Stoll, 1940; Boullier and Robert, 1992]. Ore bearing fluid is usually introduced only during part of the fracturing history, so that much of the composite vein system consists of barren minerals [Newhouse, 1940; Stoll, 1940; Knopf, 1929]. This has major implications for predicting ore grade based on a geostatistical analysis of vein geometry. Ore grade is often not strongly correlated with vein system thickness, a hypothesis that has been borne out by bitter experience [Knopf, 1929; Ray, 1954]. Recently, however, Sanderson et al. [1994] have found empirical relationships between ore grade and the distribution function describing cumulative vein thickness. In that study, high ore grade is associated with zones having large numbers of thick veins relative to thin veins. An interpretation of this observation is that the large-thickness veins must also be longer and therefore more likely to be interconnected hydraulically with remote sources of ore-bearing fluids.

The origin and description of ore bodies and procedures for interpolation and extrapolation of ore grade could be significantly advanced if the flow properties of fracture and fault intersections and the evolution of fracture permeability during dissolution and precipitation were better understood. Part of the problem is our tendency to see veins only in cross-section across the thickness (smallest dimension) of the fracture, when much deposition may be occurring along the width and breadth.

Geothermal reservoir production Natural geothermal systems involve the same processes responsible for formation of ore deposits, albeit currently active ones. Fluids become chemically active and both dissolution and precipitation are prevalent. For example, the cooling of geothermal waters during heat extraction and conversion to energy, whether from natural or hot dry rock geothermal systems, can result in sudden mineral precipitation and scaling and plugging of the plumbing at the power plant. The large-scale dissolution of minerals due solely to this type of human activity was documented during initial tapping of heat by pumping fresh water into a system of natural and induced fractures in hot dry rock at the U.S. Dept. of Energy Fenton Hill, New Mexico pilot study. Dissolution and precipitation in active hydrothermal systems have been simulated on a grand continuum scale to determine likely sites of alteration zones [Xu and Preuss, 2001]. However, these studies - like similar studies on non-reactive flow in fractured rock using continuum approaches - do not adequately consider the inhomogeneous nature of fracture permeability and flow.

Geothermal systems are often fracture dominated, and understanding where the most permeable zones are and how these change through time are important considerations for the development and production phases of the geothermal system. The U.S. Dept. of Energy maintains a research program on geothermal energy and this program has supported many studies on fracture-dominated geothermal systems. Currently, this program maintains a repository of drill core from the Awibenkok Geothermal Field in Indonesia purchased from Unocal, which is used for the study of geothermal systems. This core is rife with altered fractures and veins - examples of geochemical evolution of permeability. Finally, of interest to scholars of 
geothermal systems and ore deposits, Fouquet et al. [1998] have documented many recently discovered examples of fractures altered through hydrothermal activity from the deep ocean at the Middle Valley Bent Hill Area. Their photographs show many examples of concentrated mineralization along fractures and fracture zones.

\section{Proposed Research}

Given this background, we had proposed to study several key factors controlling the character and evolution of fracture system permeability and transport processes. We suggest that due to surface roughness and the consequent channeling in single fractures and in fracture intersections, the tendency of a fracture system to plug up, remain permeable, or for permeability to increase due to chemical dissolution/precipitation conditions will depend strongly on the instantaneous flow channel geometry. This geometry will change as chemical interaction occurs, thus changing the permeability through time. To test this hypothesis and advance further understanding toward a predictive capability, we endeavored to physically model and analyze several configurations of flow and transport of inert and chemically active fluids through channels in single fractures and through fracture intersections. This was an integrated program utilizing quantitative observations of fractures and veins in drill core, quantitative and visual observations of flow and chemical dissolution and precipitation within replicas of real rough-walled fractures and fracture intersections, and numerical modeling via lattice Boltzmann methods.

\section{Relationship to DOE/OBES mission}

Aside from the importance of this project to the specific scientific and engineering issues just described, the work is also of relevance to several DOE Office of Basic Energy Sciences Geosciences Program primary interests. Currently the DOE/BES web site:

www.science.doe.gov/bes/geo/Description/description.html

emphasizes interest in the following areas:

- Geochemistry of Mineral-Fluid Interactions

- Rates and mechanisms of reaction at the atomistic/molecular scale

- Coupled flow and reactivity in porous and fractured rocks

- Isotopic tracking of mineral-mineral and mineral-fluid processes

- Geophysical Interrogation of the Earth's Crust

- Indirect determination of geologic structure and rock properties

- Collection and analysis of seismic and electromagnetic data

- Geophysical signatures of fluids and fluid-bearing reservoirs

- Basic Properties of Rocks, Minerals, and Fluids

- Analysis of multi-phase, heterogeneous, anisotropic systems

- Determine physical, chemical, mechanical properties

- Analysis of rock deformation, flow, fracture, and failure

- Prediction of fluid transport in large-scale geologic structures 
- Analytical Instrumentation and Computational Methods

- High-resolution geophysical imaging and inversion tools

- Angström-scale resolution in analysis of heterogeneous minerals

- Advanced computational modeling and algorithm development

Our project deals with aspects important to each of the four main areas, either by providing new physical understanding of specific processes or by providing data or models useful in parameter and modeling studies.

Relationship to other projects funded by DOE In the 2004 Basic Energy Sciences, Chemical Sciences, Geosciences, and Biosciences Division Research Program Summaries we find the following list of closely related projects:

- The Physics of Two-Phase Immiscible Fluid Flow in Single Fractures and Fractured Rock. Lead Investigator: Harihar Rajaram, University of Colorado. The objective of this research is to develop quantitative understanding of the critical processes controlling two-phase flow and transport in rock fractures, with potential applications in radioactive waste isolation, $\mathrm{CO} 2$ sequestration and petroleum engineering.

- Critical Chemical-Mechanical Couplings that Define Permeability Modifications in Pressure Rock Fractures, Lead Investigator: Derek Elsworth, Pennsylvania State University. This work examines and quantifies the processes controlling rapid changes in the transport characteristics of natural fractures due to precipitation and dissolution mediated by coupled thermal-mechanical-chemical (TMC) effects.

- Fluid and Particulate Transport in Self-Affine Fractures, Lead Investigator: Joel Koplik, City University of City College, New York. They study the motion of a passive tracer in fluid flow in geological fractures with self-affine fractally rough walls by laboratory experiments on epoxy casts of natural fracture surfaces, coupled to numerical simulation using the lattice-Boltzmann method.

- Evolution of Surface Morphology During Dissolution of a Rough Fracture, Lead Investigator: Anthony Ladd, University of Florida. A fundamental understanding of the role of fractures, and the effects they have on fluid flow, solute transport and mechanical properties, is an essential component of theoretical models of geological systems. In particular, $\mathrm{CO}_{2}$ sequestration will require predictive models for the effects of fracture on the overall permeability of rock-fluid systems, and more importantly, how this fracture permeability evolves with time. The foundation of their investigation is the use of a very efficient numerical simulation of fluid flow in irregular geometries.

- Predicting Fracture Porosity Evolution in Sandstone, Lead Investigator: Stephen Laubach, Bureau of Economic Geology, University of Texas. The goal of this research is to develop an understanding of how fracture growth and diagenetic alteration interact to systematically create and destroy fracture porosity.

- Colloid Transport in Unsaturated Fractured Rocks and Porous Media, Lead Investigator: J. Wan, Lawrence Berkeley National Lab. This project involves research towards understanding vadose zone colloid transport in a thorough manner, encompassing both porous media and fractures, and accounting for differences in surface chemistry of different types of colloids.

- Reactive Transport of $\mathrm{CO}_{2}$ Rich Fluids, Lead Investigator: Russell L. Detwiler, Lawrence Livermore Natl Lab. By integrating experimental and computational techniques, they explore the processes that lead to the geochemical alteration of fracture permeabilities over a range of scales. 
- Two-Phase Immiscible Fluid Flow, Lead Investigator: Robert Glass, Sandia Natl Lab/Albuquerque, The objective of this research is to develop quantitative understanding of the critical processes controlling two-phase flow and transport in rock fractures, with potential applications in radioactive waste isolation, $\mathrm{CO}_{2}$ sequestration and petroleum engineering.

- Integrated Studies of Coupled Flow, Reaction, and Diffusion in Earth's Crust, Lead Investigator: Danny Rye, Yale University. This project involves research to improve understanding of crustal processes of fluid / rock interaction through an integration of modeling, field observations, and laboratory analysis; to develop new conceptual and computational models of coupled fluid flow and chemical reactions of geologic environments; to collect and interpret stable isotopic and geochemical field data at many spatial scales.

Our project differs from other projects on fracture hydrology funded by DOE and others in several ways. Ours is an integrated program unique in utilizing quantitative and visual observations of flow and chemical dissolution and precipitation within idealized physical models, replicas of real rough-walled fractures, fracture intersections, and fracture networks, numerical modeling via lattice Boltzmann methods, and quantitative observations of fractures and veins in outcrops and drill core. Each step in the study is designed to build and test hypotheses. This represents a joint project among staff at New England Research and Sandia National Laboratories. We divide the tasks by the expertise and available facilities at each organization.

\section{Techniques Used and Resources Applied}

- A non-contacting laser surface profiler was built allowing detailed measurements of the topography of fracture surfaces.

- Natural fractures in rock were collected for use in laboratory flow experiments.

- Techniques were developed to make plastic replicas of these fractures. The surface topography of the fracture surfaces was characterized with the profilometer.

- The plastic replicas were machined into samples allowing fluid flow and transport measurements to be made.

- Nuclear Magnetic Resonance Imaging was used to quantify the character of fluid flow channels in the fracture plane and their relation to surface roughness.

- Computer codes for modeling flow, solute transport, solute mixing, and precipitation and dissolution within fractures and fracture intersections. The lattice Boltzmann (LB) modeling codes used for this project were developed by Sandia National Laboratories and the underlying theory, mathematical models, key assumptions, verifications, and hardware requirements are documented in Stockman et al. [1999]

\section{Summary of Results}

\subsection{Mixing in Natural Fracture Intersections}

Abstract Laboratory experiments of flow through intersecting natural fractures show that solute transport deviates significantly from predictions of two-dimensional streamline routing through parallel plate intersections. Surface roughness in intersecting fractures causes two major discrepancies between mixing experiments and the parallel plate predictions. First, the fluids exiting the intersection are not uniformly 
mixed, but consist of ribbons with varied solute concentrations which tend to follow streamlines in the flow. Some of these streams maintain a nearly pure inlet fluid composition as they exit the intersection and traverse the outlet fracture. Second, when the outlet fluids are collected and homogenized, we find that this complex redirection of streamlines within natural rough-walled fracture intersections results in more total mixing than is predicted by the parallel plate model.

Sample Description Transparent plastic replicas of natural rock fracture intersections were made for visual study of transport processes. We produced replicas by first making silicone rubber molds of each of the 4 separate rock pieces comprising an intersection, and then casting epoxy resin into the molds. The final replicas reproduce the roughness features of the original rock at scales better than $10-100 \mu \mathrm{m}$. Inlet and outlet manifolds were fitted to the specimens to uniformly distribute flow along the fractures.

Two distinctly different natural rock fracture intersections were studied. One sample, a siltstone from Cedar Crest, New Mexico, contains a natural joint with a calcite coating and a slickenside texture. An orthogonal tension fracture was introduced by splitting the sample between wedges. The two fractures thus meet and form an intersection. The slickenside fracture has anisotropic roughness which gives rise to strong channels of flow, while the tension fracture roughness is more isotropic. During assembly, the tension fracture surfaces were misaligned slightly to give each fracture approximately the same average aperture and flow capacity. Figure 5 shows aperture fields for the fractures of the slickenside sample as determined by image analysis.

The other sample consists of two intersecting natural fractures in a fine-grained schist from Northfield, Vermont. The fracture in the plane of cleavage (schistosity) is slightly smoother and more planar than the other cross-cutting fracture.

Experimental Method In the experiments two adjacent fracture legs act as flow inlets and the other two legs act as flow outlets. Samples are initially saturated with pure water and flow is single phase. Homogeneous dye solution flows into one inlet, and clear water into the other. A constant head system maintains flow through the sample by gravity. Inlet and outlet flow rates are measured and controlled.

The electrical conductivity of the two outlet solutions was measured continuously. This gives the amount of average mixing that has occurred, because the two inlet solutions have markedly different conductivities. We use distilled water as one inlet fluid and dye solution made with tap water as the other.

Two CCD cameras took still pictures of the steady state dye distribution within each intersecting fracture. Image analysis gives quantitative measures of both the aperture distribution and dye concentrations within the sample. The Lambert-Beer law relates the monochromatic absorbency of dye to the concentration and aperture at that pixel as:

$$
A=\mu C d=\ln \left(I_{H_{2} O} / I_{d y e}\right),
$$

where $A$ is absorbency, $I_{\mathrm{H}_{2} \mathrm{O}}$ and $I_{d y e}$ are the measured pixel intensity (gray level), $\mu$ is the extinction coefficient of the dye, $C$ is dye concentration, and $d$ is aperture. Following a calibration step, application of Equation (3) for the images produces quantitative local variations in dye concentration.

The flow rate at each inlet of the samples was fixed and the flow rate for each outlet leg was varied from 0 upward to the maximum. The average Reynolds number at the maximum outlet flow rate was calculated to be a maximum of 2.4 for all tests. Flow rates were chosen to give Reynolds numbers that insure laminar flow through the sample without large inertial flow effects, while at the same time maintaining high Peclet numbers allowing us to ignore molecular diffusion as an important mechanism for mixing. Streamline routing is therefore the appropriate end-member model for comparing our results. 
Observations Figure 6 shows typical images of steady state dye concentration in the outlet legs of the siltstone sample. Images from the schist sample show similar features. All observable mixing occurs in the intersection. Fluid exiting the intersection contains "ribbons" of various distinct solute concentrations which follow streamlines in the flow. Adjacent streamlines do not mix but retain constant concentrations along the outlet fractures, which is consistent with steady state flow measurements at high Peclet numbers. Tortuous flow and channeling, as delineated by the dye ribbons, is expected for single fracture flow. However, solute transport through the intersection differs from parallel plate streamline routing, because both inlet fluids (dye and pure water) are observed to exit through both outlet legs, and because the dye concentration is not spatially uniform.

Many dye ribbons are of intermediate concentration, but nearly pure streams of dye or water can cross right through the intersection, apparently unaffected by the perpendicular fracture. When we look directly into the intersection this behavior is explained: fluid from one inlet fracture can "push" its way up into the adjacent inlet fracture a small amount, blocking local flow from the other inlet. Once this happens, fluid flowing from this place into both outlet legs will remain pure. This "intersection blocking" is seen in both samples.

Quantitative mixing results are determined from the outlet fluid electrical conductivity. When all flow rates are equal (flow fraction $=0.5$ ), outlet concentrations are $25 \%$ and $75 \%$, instead of $0 \%$ and $100 \%$ as predicted by streamline routing for in parallel plates. Streamline routing predicts that at least one outlet leg will only contain pure fluid from the adjacent inlet leg. Our experimental observations are inconsistent with this prediction.

Intersection geometry and fracture roughness have pronounced effects. Even a small offset of one fracture across the other is observed to have a large effect on mixing. When roughness is anisotropic, flow channels apparently correlate across the intersection and preferentially allow undiluted fluid to flow straight through the intersection by intersection blocking.

Conclusions We observe that solute transport through natural fracture intersections with negligible molecular diffusion can differ considerably from mixing in parallel plate intersections. Aperture variability in the intersecting fractures due to surface roughness allows pure fingers of one solution to cross straight through an intersection, by blocking flow from the adjacent inlet. Our data imply that while streamline routing may hold locally at the intersection, three dimensional flow rate variations can lead to increased mixing when outlet dye concentrations are averaged over the length of the intersection. Our results show that parallel plate streamline routing may not be an accurate advection dominated end-member mixing model for intersecting natural fractures.

\subsection{Mixing in Synthetic Fracture Intersections}

Abstract To better quantify the observations of mixing in the natural fracture intersections, we performed laboratory experiments of mixing of two miscible fluids within an artificial fracture intersection. Using insight from these experiments, we developed a numerical model of mixing based on local application of streamline routing within the irregular aperture distribution composing the intersection. This model shows good agreement with the laboratory experiments, both in the amount of average mixing and in the spatial distribution of dye streamlines. This model also shows how flow channelization leads to enhanced average mixing. Through parameter studies, we explore the effects of aperture statistics on mixing and find that mixing is significantly affected by how well apertures correlate across the intersection, especially as apertures are closed.

Experimental Setup Using pieces of textured and flat glass we constructed a model fracture intersection for laboratory study (Figure 7a). The specimen consisted of four fracture legs, approximately $6 \mathrm{~cm}$ square, 


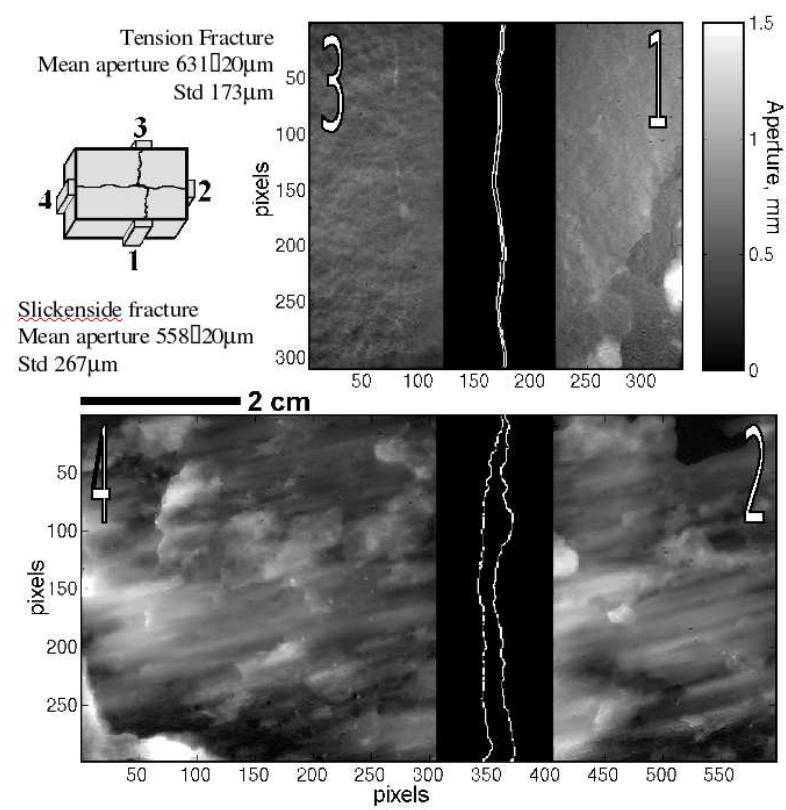

Figure 5: Siltstone fracture apertures. Direct view of the intersection is obscured by the orthogonal fracture and flow manifolds, so aperture fields do not extend to the intersection. Traces of the orthogonal fractures are indicated. Note the tension fracture offset across the slickenside fracture. The upper corner of leg 2 shows a blob of epoxy which dripped a small way into the aperture during fabrication. The 3D sample schematic shows the offset as well as flow manifolds. The schematic view is primarily from the top, while aperture images are side views of the sample. 

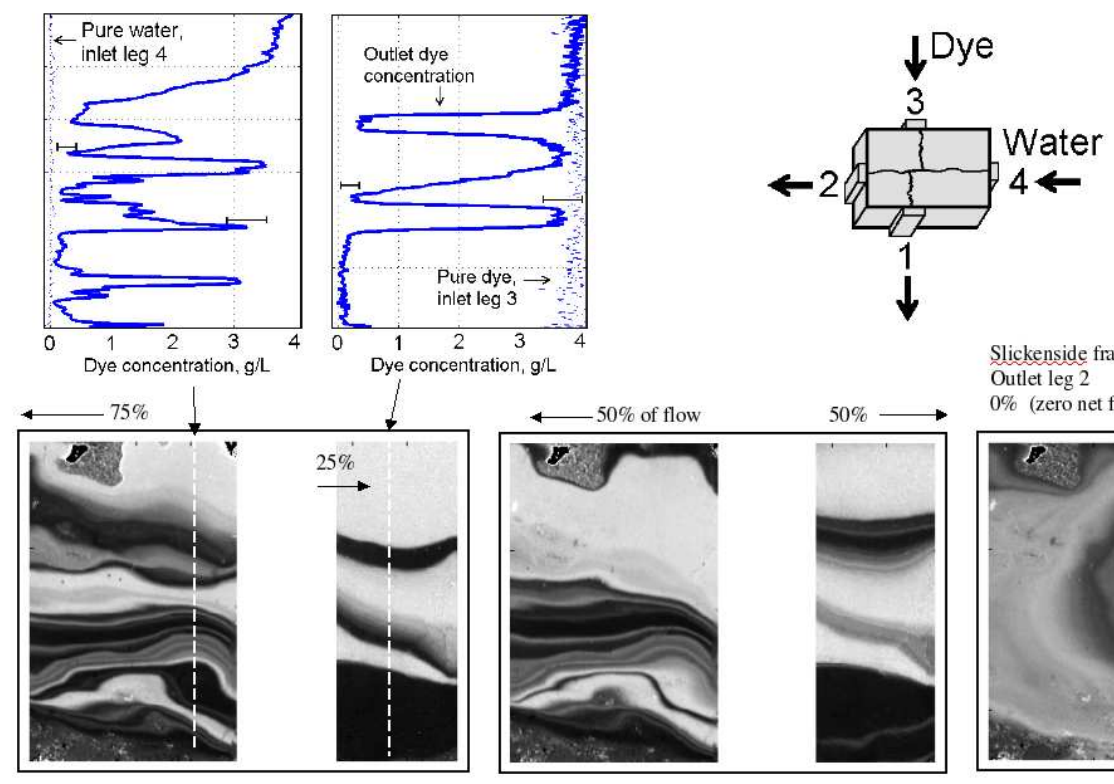

Slickenside fracture Outlet leg 2

$0 \%$ (zero net flow)

Tension Fracture Outlet leg 1 $100 \%$ of flow

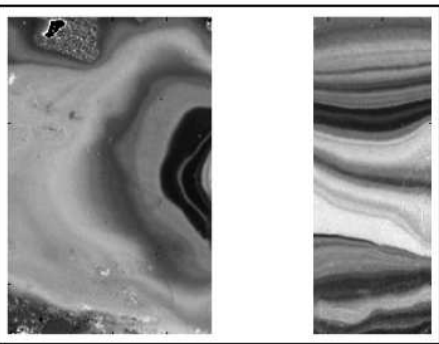

Figure 6: Dye concentration in the siltstone sample of Figure 5. The two inlet legs $(3,4)$ each receive the same input flow rate and the two outlet legs $(1,2)$ receive a varying proportion of the output flow. The three lower images show the dye concentration in outlet legs 2 (left) and 1 (right) as the proportions of the total outlet flow rate for these two legs is set at $75 \%-25 \%, 50 \%-50 \%$, and $0 \%-100 \%$, respectively. Flow is from the center if each image (intersection) to the outside. As in Figure 1, direct view of the intersection is obscured by the manifolds. The graphs in the upper left compare specific dye concentration profiles to the pure inlet fluids. 


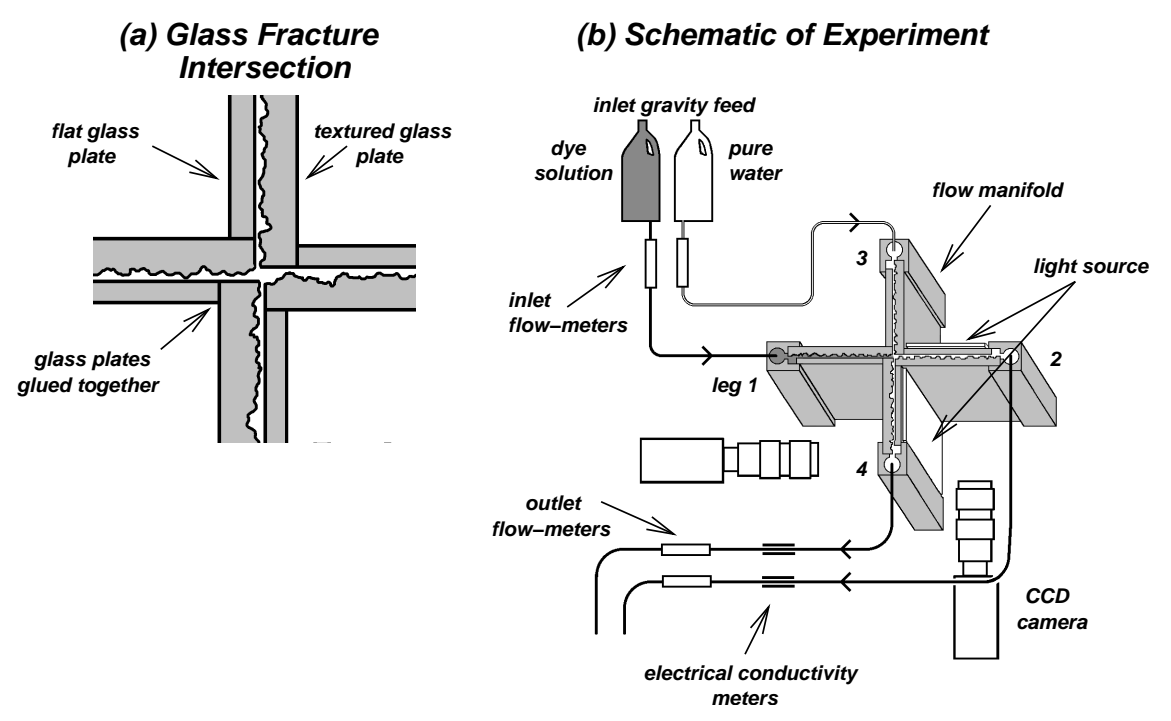

Figure 7: Fracture intersection flow cell. (a) Closeup of the sample intersection, showing how the textured and flat glass sheets were aligned and glued together. (b) Schematic of experiments, showing sample inlets and outlets configured for a continuous flow experiment. Digital cameras provide aperture and dye concentration data at the outlets during the experiments.

meeting at right angles. Flow manifolds on each of the fracture legs were designed to distribute fluid along the edge of each aperture. Apertures for opposite (parallel) legs did not consistently correlate across the orthogonal fracture, because the four textured glass pieces were cut from different places on the glass sheet.

The experimental setup for measuring aperture fields, solute concentration, and flow was the same as we described before. Two sample legs acted as flow inlets, one for dye solution and one for distilled water (Figure 7b). Inlet and outlet flow rates were controlled and measured using a gravity-fed system fitted with flow meters. All flow and transport measurements were collected at steady state conditions. Average outlet fluid concentrations were determined by measuring the outlet fluid electrical conductivity, because the dye itself conveniently acts as an electrolyte. Conductivities were measured after the fluid exited the outlet flow manifolds. Two CCD cameras provided digital images of flow in the fracture during the experiment. Image analysis gave quantitative measurements of both the aperture distribution and dye concentrations within the sample.

Apertures were measured right up to the intersection for each of the four textured glass pieces before assembling the sample. Figure 8 shows an aperture field determined for one of the four legs. Semivariogram analysis of the aperture field shows that at the sample scale the aperture can be treated as a homogeneous random field. Knowing the detailed aperture of each fracture leg in this manner has allowed us to model fluid flow and mixing using a Reynolds equation model.

Numerical Model The Reynolds equation describes two-dimensional flow between rough surfaces with smoothly varying aperture. It has been widely used to model flow in rough-walled fractures. The Reynolds equation assumes that the cubic law for parallel-plate flow holds locally in the variable-aperture fracture.

For our studies, we solved a finite-difference form of Reynolds equation by using the iterative method of successive over-relaxation. To model intersecting fractures, flow was solved for simultaneously in two separate aperture fields. The aperture fields were assumed to intersect along a single column of pixels from each fracture. Source and sink terms were used along this column to create an intersection and flow was forced to be conserved around and through the intersection. 


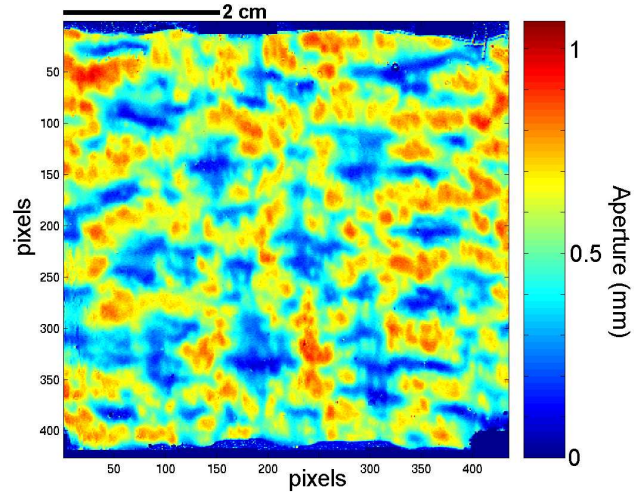

Figure 8: Aperture field for leg 4 of the textured glass measured by dye absorbency as described in the text. The glass we used had anisotropic roughness. The textured glass, and thus the aperture, has some patterning leading to a slight anisotropy in roughness and flow properties.
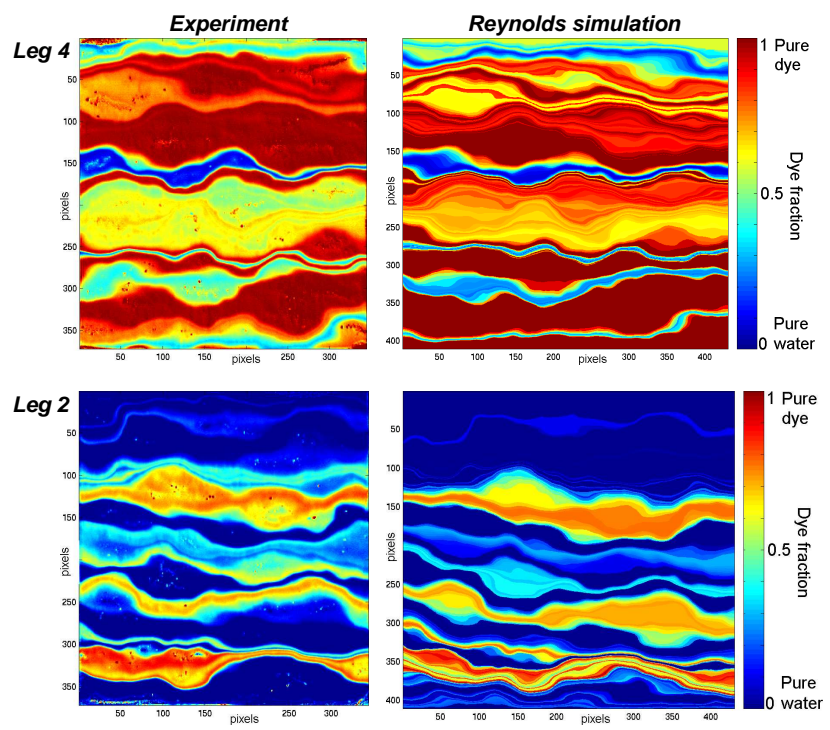

Figure 9: Experimental and simulated outlet concentrations, continuous test 1. Average flow rates are equal for both outlet legs (flow fraction 0.5). The quantitative image analysis methods used to measure experimental dye concentrations are described in the text. Flow is from left to right in each image. The experimental concentration images cover a slightly smaller subset of area than that shown for simulations. 
The numerical solution to the Reynolds equation provides a description of flow rates and channel geometry in and around the intersection. To model steady-state solute transport and mixing, we further assumed that parallel-plate streamline routing held locally everywhere within the intersection. Parallel-plate streamline routing (Equation 1) was applied at each intersection pixel using local flow rates to determine solute concentrations flowing into each outlet leg, for both continuous and discontinuous flow. Diffusion was not included, so simulations should correspond to the high Peclet number limit of streamline routing behavior.

Simulations were designed to match the experimental conditions of the laboratory measurements. Simulations were run using the full aperture fields determined by image analysis for the laboratory sample.

Conclusions When flow is continuous, mixing in rough-walled synthetic fractures is considerably enhanced compared to parallel-plate streamline routing predictions. Outlet concentrations are not uniformly mixed within rough-walled intersection outlet legs, but consist of ribbons or streamlines of solute. Average mixing for discontinuous flow is observed to match parallel-plate streamline routing predictions reasonably well. However, outlet fluids still show considerable spatial concentration variations.

Qualitatively, observations of mixing behavior in the textured glass sample studied here are consistent with the experimental results from the natural intersections. As evidenced in all laboratory experiments and in the numerical studies, mixing in rough walled natural and synthetic fractures, where flow is three dimensional, shows marked differences from the two dimensional parallel-plate streamline routing predictions. Outlet concentrations are not uniformly mixed within rough-walled intersection outlet legs, but consist of ribbons or streamlines of solute.

Experimental results of mixing can be well matched by numerical simulations when the aperture fields of the intersecting fractures are explicitly known (Figure 9). In these simulations, the fracture intersection was assumed to behave like a large number of individual parallel-plate intersections. This mechanism is sufficient to predict average mixing as well as the spatial distribution of solute streamlines, provided that advection dominates over diffusion. In contrast, parallel-plate streamline routing accurately describes mixing in 2D, but underestimates mixing when applied to an entire 3D fracture intersection.

Numerical simulations show that mixing approaches parallel-plate streamline routing predictions as fracture apertures are opened. As apertures close, flow at the intersection becomes increasingly concentrated within the remaining interconnected open aperture channels. Mixing tends to increase with closure, but whether a particular flow channel correlates across (opposite outlet) or around (adjacent outlet) can cause significant variations in mixing from intersection to intersection, even if the fracture aperture statistics are the same.

Fine-scale surface roughness has only a small effect on mixing compared to the longer wavelength features which create flow path geometry. Anisotropic roughness affects flow channeling in interesting ways. Longitudinal roughness tends to enhance channeling, slightly increasing mixing. Transverse roughness decreases channeling in open fractures, but tends to increase channeling and mixing in tight fractures.

\subsection{Mixing in Fracture Networks}

Abstract We use a numerical network model to explore intersection mixing effects on networks. Our numerical model only considers the complicating factor of rough walled surfaces on dispersion in a lattice network of identical intersections. The fracture apertures used for network flow simulations were determined experimentally for the textured glass laboratory sample of a single fracture intersection. The Reynolds equation for two-dimensional flow through variable aperture fields is solved for two intersecting aperture fields. To make a network, the flow vectors determined for the intersecting fracture pair are replicated to each intersection of the entire network. Adjacent inlet and outlet flow vectors are handled in a consistent fashion. Next, steady state solute dispersion through the network is solved by tracing the concentrations of flow streamlines from the outlets of one intersection to the inlets of the next. The model shows the lateral 


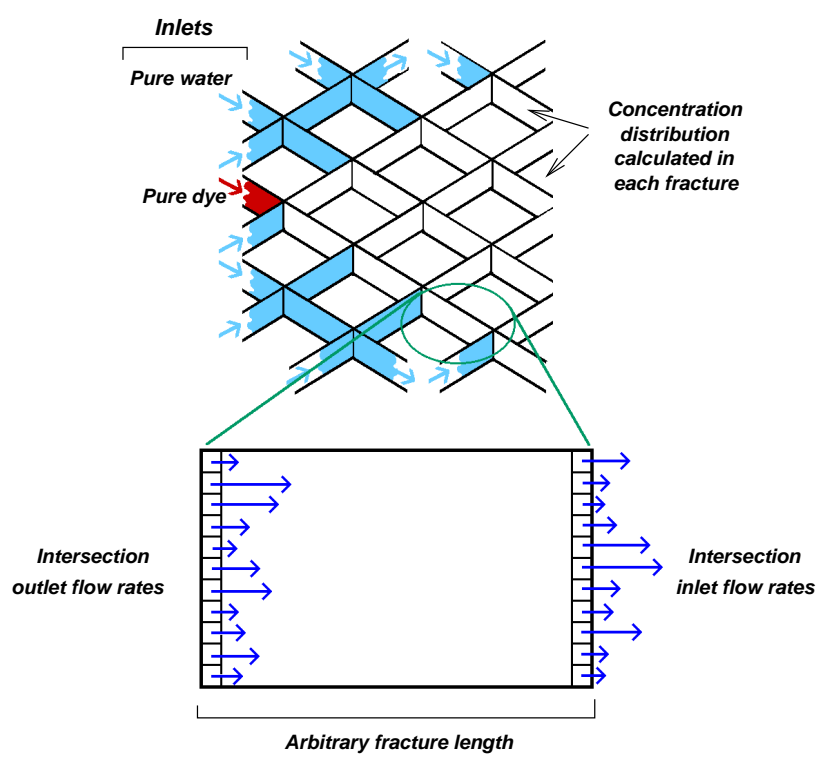

Figure 10: Construction of the network model. A regular lattice of fractures is constricted, with dye introduced at the upstream end of one. Dye dispersion is tracked by assigning a distribution of intersection inlet and outlet flow rates sampled from the textured glass Reynolds simulations and using a point by point streamline routing model along each intersection.

dispersion within the network and the spatial streamline concentrations in each fracture, but not the time dependence of transport. We compare the numerical results to more idealized predictions of dilution and dispersion in the essentially two dimensional case of flow through parallel plate lattice networks.

Network Model We present simple numerical simulations of steady state solute transport through a network of fractures with known inlet and outlet flow rate distributions. We assume a regular lattice network of fractures with equal hydraulic conductivity. Each fracture in the network is statistically equivalent to the textured glass fracture studied previously. The average flow rates through each fracture are the same yet the flow in and out of the intersections are sampled from a distribution of possibilities.

To obtain the inlet and outlet flows, the spatial distribution of flow rates through the glass fracture was determined using the Reynolds equation. At each column of pixels perpendicular to the flow, this results in two grids of flow rate vectors, qx (parallel to pressure gradient) and qy (perpendicular to pressure gradient). The qx vectors sum (along each column) to give the total simulated flow rate through the individual fracture. The grid size of the Reynolds equation flow simulations for individual fractures used to populate the network model were 300 rows by 600 columns.

Each intersection of the regular lattice network consists of two inlets and two outlets. For an individual intersection, the spatial distribution of flow rates is set as follows: First, two qx flow rate vector columns are chosen at random from the 600 columns of the single fracture simulation. These define the distribution of inlet flow rates into the intersection. Then, two different qx flow rate columns are chosen to approximately determine the amount of total flow at a given location that reaches each outlet. Each intersection of the fracture network is populated with flow rates in this manner. Thus each intersection represents a statistical realization of the possibilities for inlet and outlet flows.

For the simulations presented here a network of 101 by 101 fractures was used, with each intersection containing 300 individual flow rate vectors at each inlet and outlet (Figure 10). For the simulations presented here, one can visualize the network as being long in the $\mathrm{x}$ and y plane (101 fractures), with a "height" of 


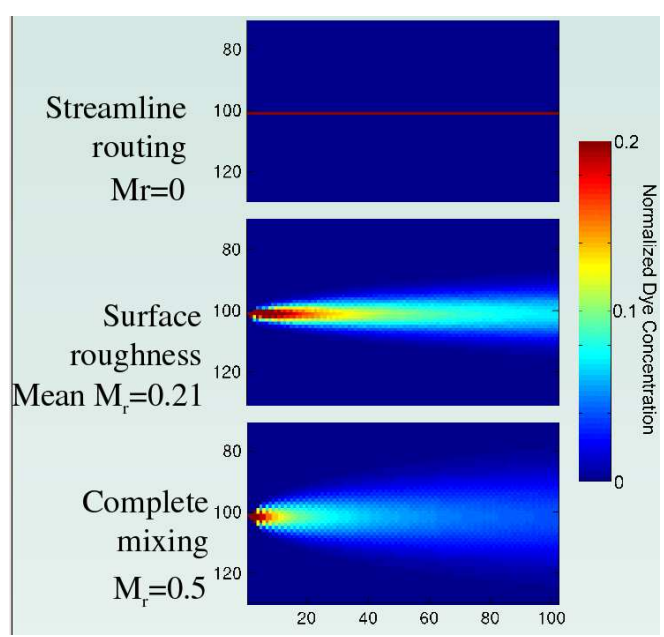

Figure 11: Examples of 2-D distributions of dye dispersing in a fracture network for three different mixing models.

300 discrete flow rates per fracture. Each fracture of the network consists only of outlet flow rates from the previous intersection, and inlet flow rates from the next intersection. The length of each individual fracture (distance between intersections) is arbitrary since only inlet and outlet flow rates are defined.

Once the network of flow rates has been defined, solute transport is calculated. One inlet fracture at the center of the grid contains pure "dye", with a concentration set to 1; all other fractures along the inlet side start with pure water, concentration 0 . There are two steps to calculating steady state dye transport through the network: First, dye must be distributed from the inlet fractures to the outlet fractures. Second, dye must be transported through the fracture, from the current outlet side to the next inlet side.

Dye is transported through an intersection by assuming that parallel plate streamline routing holds locally, at each of the 300 vectors that define flow into and out of the intersection. This assumption has previously been shown to adequately describe intersection mixing when the vector flow rates through the intersection are known. Solute transport through the intersection is therefore based only on advection, and does not include molecular diffusion. As dye moves from one intersection through a fracture and is mixed with fluid at the next, the total mass of dye is conserved.

The result of intersection mixing is typically a distribution of dye concentrations along the outlet flow vectors (Figure 11).

Conclusions Several cases were studied using this network model: parallel-plate mixing using both 2-D streamline routing and complete mixing models, 2-D mixing through rough fractures (by using the average mixing ratio observed in the glass fracture experiments), and 3-D mixing - using the statistical distribution of flow rates as just described (Figure 12). We find that the results for rough surfaces fall between the idealized mixing models. Surface roughness increases steady state solute dilution and dispersion above the simple parallel plate model. A goal of this research was to compare dilution and dispersion through a network with surface roughness to parallel plate fracture networks. Significantly, we find that dispersion in 2D parallel plate networks matches that in our networks as long as the mean intersection mixing ratio is known. This is significant because it implies that understanding the dynamics of a "representative" fracture intersection can allow prediction of the larger behavior of the network. 


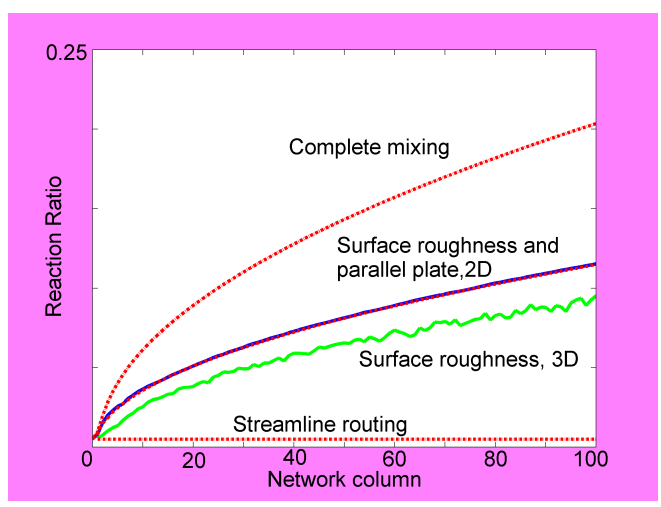

Figure 12: Plots of reaction ratio (dye dilution index) for various mixing models in the network simulation. Reaction ratio $=0$ for zero dilution of dye, reaction ratio $=1$ for maximum dilution.

\subsection{MRI Studies of Mixing Within Intersections (Collaborative Project)}

Magnetic resonance imaging (MRI) experiments were done on two intersecting fracture models. Initially we worked with an idealized model, consisting of two parallel-plate fractures, $8 \mathrm{~cm}$ long, $5.5 \mathrm{~cm}$ high, 0.2 $\mathrm{mm}$ apart, and intersecting at right angles. The second model consisted of an epoxy replica of the same slickensided siltstone rock sample studied earlier by optical/dye methods.

In our experiments we had the continuous flow geometry with two adjacent inlets and two adjacent outlets. On both the inlet and the outlet sides valves were inserted so that the flow in each branch could be independently controlled. The flow was driven by a constant pressure head.

The MRI experiment was done in a $31 \mathrm{~cm}, 1.9$ Tesla magnet with an attached imager/spectrometer system. All the images and flow measurements were done with a spin echo pulse sequence with an echo time of approximately $10 \mathrm{~ms}$. The goal of the study was test the ability of MRI methods to obtain structural (aperture geometry) information, to make local velocity measurements, and to study mixing behavior. The techniques for structure and fluid flow measurements using MRI as used in this study are described further by Caprihan and Fukushima [1990]; Callaghan [1991]; Seymour and Callaghan [1997].

Structure Imaging Three experimental methods (2D, 2D multi-slice, and 3D) were done to image the fluid within the fracture models. A horizontal 2D slice was taken, which is like a top-view and gives the geometry of the fracture intersection (see Figure 13a for the siltstone rock fracture model). The slice was $2 \mathrm{~mm}$ thick and had an in-plane resolution of $0.5 \mathrm{~mm} \times 0.5 \mathrm{~mm}$. This horizontal slice then served as a scout image to select a slice along the fracture and make its in-plane image (Figure 13b). In this case the slice thickness was $5 \mathrm{~mm}$ and the in-plane resolution was $0.5 \mathrm{~mm} \times 0.5 \mathrm{~mm}$. The thickness of the slice was chosen to be sufficiently wide to include the fracture. One has to be careful in the case of the rock fracture model to include the fracture within the slice because it does not lie all in one plane.

Good structural images of the fracture (i.e., the location and volume of fluid within the aperture) are obtained away from the intersection. However, at the intersection the signal from the orthogonal fracture gets projected onto the image. This leads to a bright line on the image, which marks the intersection and leads to loss of information close to the intersection. The only way to avoid this information loss was to do a $3 \mathrm{D}$ experiment or a multi-slice $2 \mathrm{D}$ experiment. We give results of such a multi-slice experiment. We used 32 horizontal slices each $2 \mathrm{~mm}$ thick to cover the sample. The sample structure can then be visualized by stacking these 32 slices. A surface rendered picture of fracture is shown in Figure 13c. This 2D multi-slice data lets us get structural information close to the intersection. 

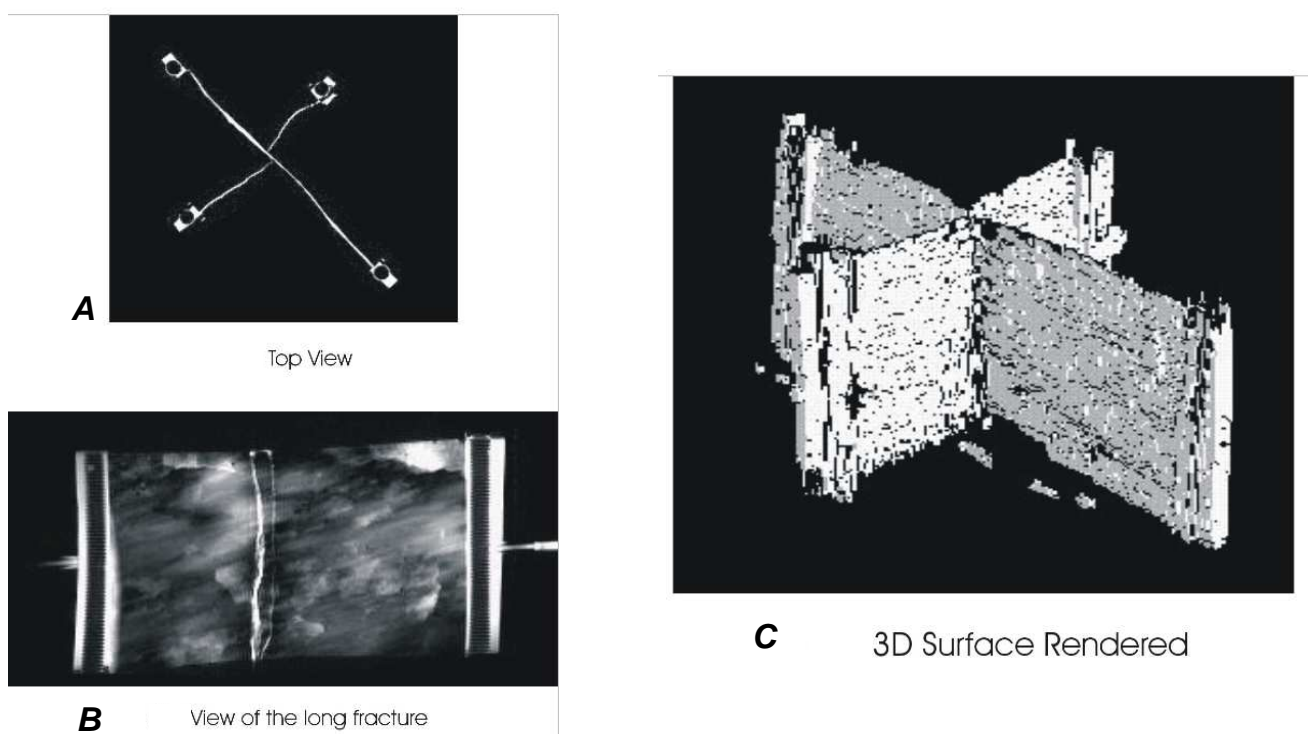

Figure 13: MRI images of fracture aperture within the siltstone rock sample.

Velocity measurements Velocity measurements were made by both the tagging method and by the phase method. The tagging method is easy to understand: the magnetic spins in the water are magnetically saturated along only an appropriate slice and an images is formed after a short time delay. Saturation destroys the magnetization and these spins appear dark in the image. The delay between saturation and imaging gives time for the spins to move, thus the image appears in a different place than expected - allowing the velocity to be inferred. The delay time has to be sufficiently long so that spins move a distance greater than the spatial resolution of the image. After saturation the spins recover their magnetization exponentially with a characteristic time constant. As time increases, the contrast between the saturated and the unsaturated region gets smaller and we will not be able to distinguish between the tagged slice and the rest of the image.

Although the tagging experiment is a quick way to measure velocities, it only gives velocity along one line in the image. We can partly reduce this problem by saturating multiple lines. However, this method can never give velocity measurements at all the points in the image.

Another technique, the phase method of velocity measurement, can give velocity measurements for all image points. The results for velocity measurement by the phase method are shown in Figure 14. We also show velocity profiles along different vertical sections in the fracture. These measurements show strong tendency for flow channeling in the fractures and in some cases, negative velocities near the intersection.

Conclusions We find that magnetic resonance imaging (MRI) is a useful technique to make velocity measurements in a parallel plate model of fractures away from the intersection. We also have reasonable success with the rock fracture model when looking away from the intersection. The methods we employ for imaging are not suitable for measuring flow properties close to and within the intersection. It would be possible to study flow close to the intersection by $2 \mathrm{D}$ multi-slice or 3D experiments. We are able to obtain structural images with these experiments but the signal loss with flow prevented us from making flow measurements. In order to look with the intersection we would need higher resolution imaging. All our experiments were done with $0.5 \mathrm{~mm}$ resolution and in some cases $0.4 \mathrm{~mm}$. A resolution of $0.1 \mathrm{~mm}$ would have given us sufficient pixels within the intersection. This kind of resolution is not possible with our present MRI system. We need higher magnetic field gradients to achieve this resolution. Although, even if we could get higher resolution, we would still require considerably greater signal averaging to get the same signal-to-noise ratio. One option 


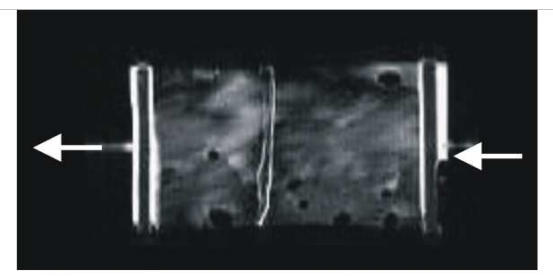

A Structure image of the rock fracture

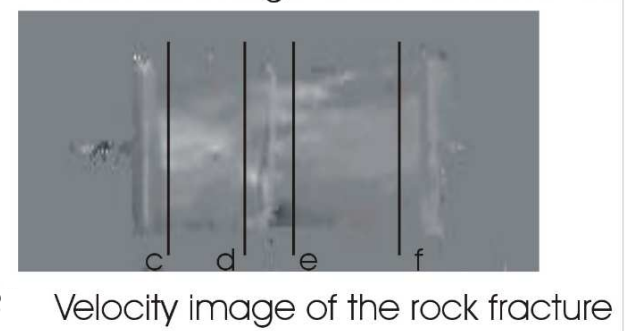

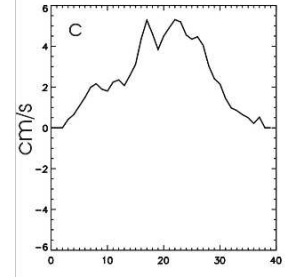
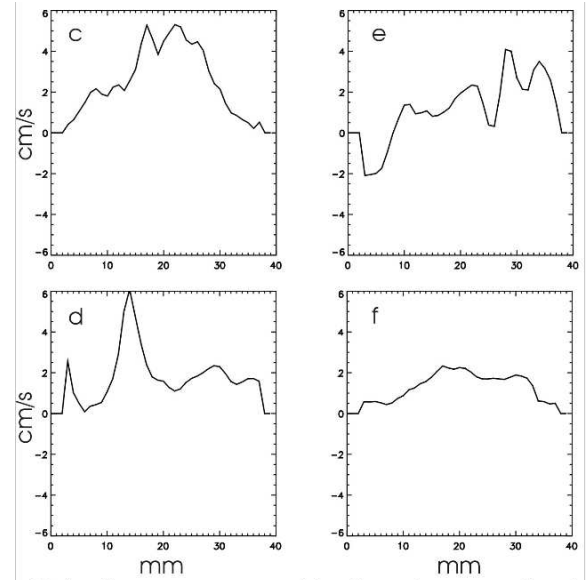
C Velocity measurement by the phase method
for the rock fracture model

Figure 14: MRI flow velocity measurements within the siltstone rock sample.

that we have not fully explored is to sacrifice spatial resolution and only measure velocity in a probabilistic sense. In other words, measure velocity histograms over larger spatial regions. These experiments might be useful in situations where fractures are complex and it is not possible to resolve them in great spatial detail.

\subsection{Formation of Fracture Networks (Collaborative Project)}

Abstract As part of this project, S.R. Brown provided technical support and acted as a faculty adviser for an M.S. graduate student thesis project at Dartmouth College. The idea linking this thesis to the DOE/BES research grant is that once the behavior of flow in fracture intersections is understood, the next step involves description of flow and channeling in fracture networks. Therefore, we need to gain fundamental understanding of the parameters controlling fracture system geometry.

Sets of parallel, closely-spaced joints in rock must arise from a system of forces or strains acting uniformly throughout the body. One possible way such a "body force" can be produced and give rise to regional joint sets in flat-lying rocks is as follows: Assume that individual layers of rock in a sedimentary sequence have a marked contrast in Young's modulus, but similar Poisson's ratio. When load is applied normal to the layers, different lateral strains between the layers result in the least stiff layers exerting tensile forces or extensile strains across each common interface with a stiffer layer. On the local level the tensile failure criterion can be reached in the stiff layers leading to the creation of a joint. When one joint is created, the stresses or strains are only relaxed locally and thus other fractures can form some small distance away.

Methods We have carried out laboratory experiments demonstrating the ability of this extensile loading mechanism to generate systematic fracture systems with a variety of characteristics (Figure 15). An aluminum loading fixture was machined to contain a thin tabular specimen of rock or plaster and two bounding layers of rubber. The sandwich of rubber and rock is loaded in a hydraulic press perpendicular to the layering. The rubber is considerably less stiff than the rock. Thus, at a given load, it expands more in the lateral direction than the rock, imparting tractions on the interface and resulting in high tensile stresses in the rock layer.

A channel in the aluminum loading fixture constrains the expansion of both the rubber and the rock in one direction only. This acts as a simulated intermediate principal tectonic stress. As the assembly is loaded normal to the "bedding", the rubber expands in the free direction, leading to the formation of multiple 


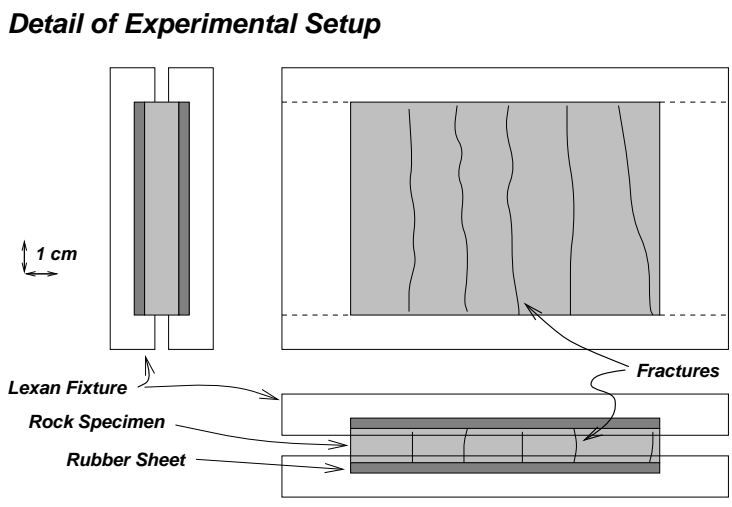

Loading Configuration

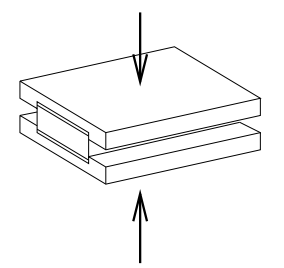

Figure 15: Experimental setup for joint system formation in layered rocks.
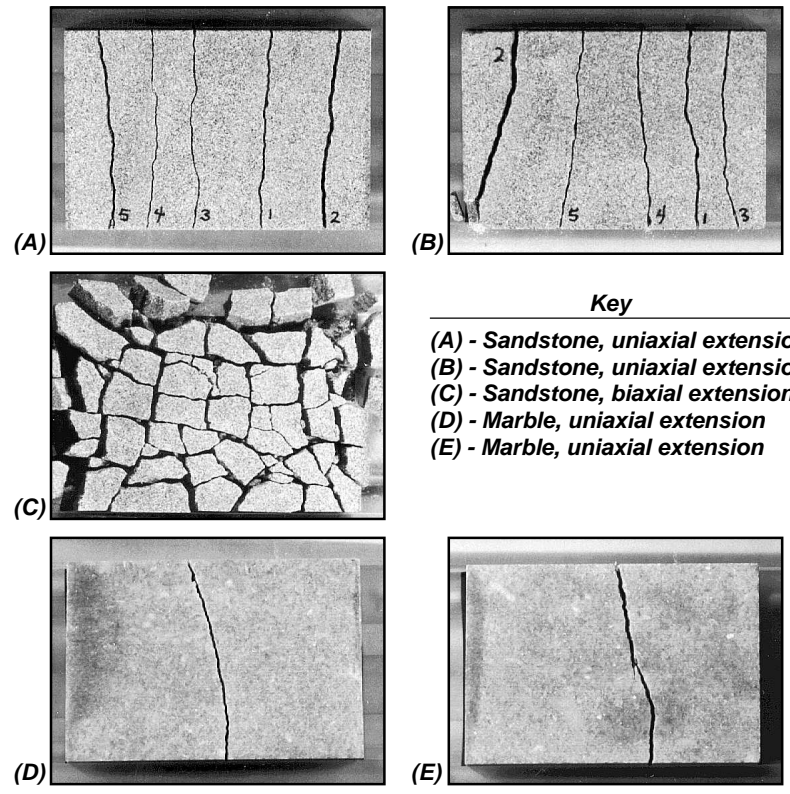

(A) - Sandstone, uniaxial extension

(B) - Sandstone, uniaxial extension

(C) - Sandstone, biaxial extension

(D) - Marble, uniaxial extension

(E) - Marble, uniaxial extension

(E)

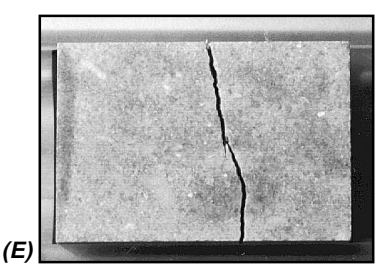

Figure 16: Joint sets formed by compression perpendicular to layering.

parallel fractures in the rock layer (Figure 16). The formation time and location of each new fracture can be observed in both the loading record and visually on the edge of the specimen.

Conclusions Parameter studies have been performed where tensile strength, maximum load, and specimen thickness were varied. There are clear trends of increasing fracture density with decreasing specimen thickness and a scaling of fracture density with maximum load relative to tensile strength. Quantitative measurements show that these experiments produce fracture densities in the range commonly observed in rock outcrops. Further experiments of this type may lead to a clearer understanding of the often-cited fracture spacing versus bed thickness relations observed in field data. Our results provide controlled experimental data against which recent theories on joint formation in layered rock can be tested and refined.

\subsection{Field Measurement of Fracture Aperture}

Abstract To enable us to tie our results to field applications, we built a portable hand-held air permeameter to be used for measurement of effective fracture flow apertures at field sites (Figure 17). The operator builds 


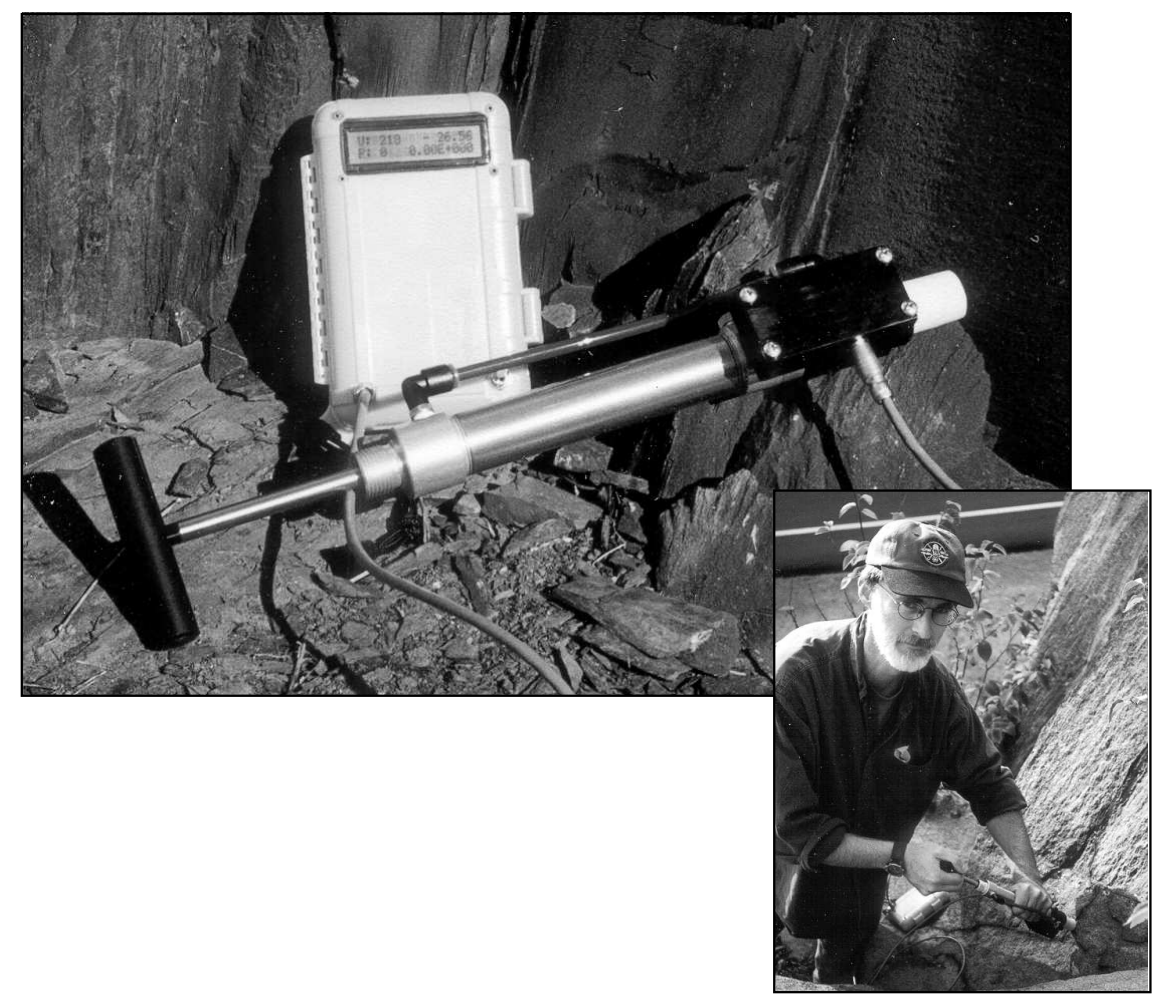

Figure 17: The hand-held air permeameter for fractures christened "TinyPerm."

pressure in a chamber with a hand pump, then discharges the compressed air into a fracture through a rubber nozzle. A micro-controller measures the time in milliseconds required for the air pressure in the chamber to drop to $1 / 2$ of a preset value. Calibrations show that this decay time can be used to accurately derive fracture apertures in the range 25-2000 microns (Figure 18). This device was used in the field to determine the variability of apertures in fracture networks, specifically around fracture intersections.

Conclusion For this project, one simple question was answered through the use of this device: Does the line of intersection of two fractures comprise a significant channel for fluid flow in its own right, above and beyond the contribution of the two fractures themselves? After repeated scoping experiments at field outcrops, no evidence was found for enhanced flow along fracture intersections above the natural variability of the individual fracture permeabilities.

\subsection{Dissolution in Fractures}

Abstract We investigated the complex active chemical interaction between pore fluid and fractures that causes the fluid composition and fracture surface topography of these systems to change over time. Our experimental model consists of flat planar gypsum samples pressed with a constant force against an inert textured glass surface. Pore fluids ranging from unsaturated to supersaturated, which are at a variety of flow rates, are introduced to one end of the sample in order to actively alter the topography of the gypsum surface. Using a laser profiler, we are able to quantitatively monitor the changing surface topography over time as it relates to the measured sample permeability and calcium saturation of the pore fluid. These methods allow us to create and analyze many features seen in natural fractures, including high-flow dissolution channels, plateaus, and caverns formed from precipitate. In addition, the laser profile of the sample surface can be 


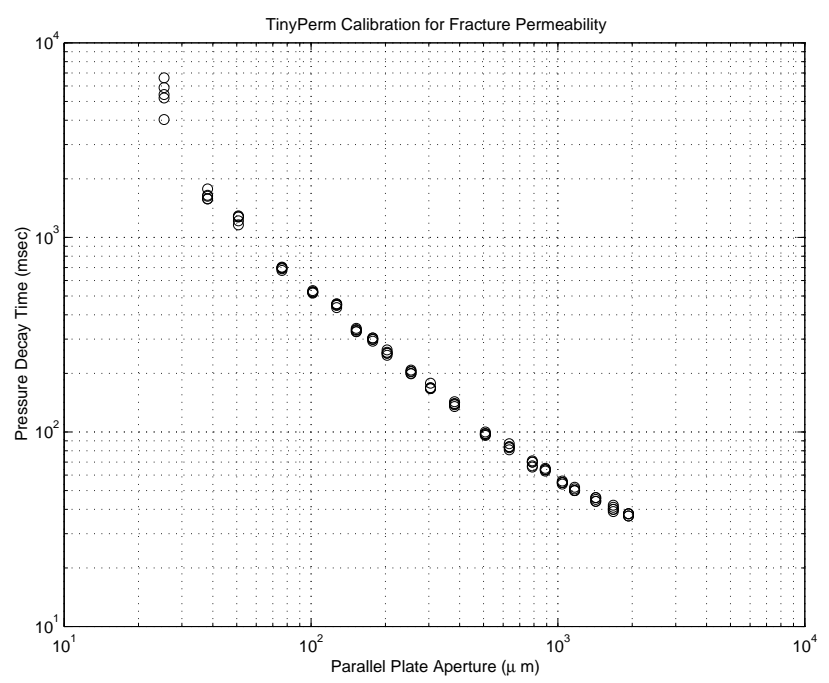

Figure 18: TinyPerm calibration relating fracture aperture to pressure decay time.

used to produce a map of aperture across the sample. Using this information, we have applied numerical modeling via finite difference and lattice Boltzmann (LB) methods to calculate pore fluid flow direction and magnitude over the entire sample surface.

Laboratory Studies In the laboratory, we directly observed chemically active flow within single fractures. We assembled laboratory apparatus and developed techniques for fracture permeability measurements under dissolution conditions. Our experimental model consists of gypsum $\left(\mathrm{CaSO}_{4} \cdot 2 \mathrm{H}_{2} \mathrm{O}\right)$ samples (dental plaster) pressed against an inert textured reference surface (either textured glass or an epoxy cast of a rough surface) (Figure 19a). Experiments with either fixed-displacement or constant normal stress boundary conditions between the two contacting surfaces can be performed. The topography of the textured surface induces heterogeneous flow along the plaster specimens (Figure 19b). Pore fluids ranging from unsaturated to supersaturated with respect to gypsum are introduced to the sample in order to chemically alter the topography of the plaster surface.

The flow system is gravity-fed with a constant pressure head or flow can be forced at a specific rate using a peristaltic pump. The specimens can range from partially- to fully-saturated. Permeability changes are monitored with a flow meter and we monitor the electrical conductivity of the outlet fluid as a proxy for calcium saturation (calibrated by selective sampling). We are able to monitor visually the state of the sample and flow system through the transparent reference surface (textured shower glass) (Figure 19d). Using a high-resolution laser profiler, we are able to monitor quantitatively the changing surface topography in 2-D as it relates to the measured sample permeability and calcium saturation of the pore fluid (Figure $19 \mathrm{c})$.

When experimental parameters (e.g., initial surface topography, flow rate history, and total experiment time) are duplicated, the topography developed on the plaster sample is reproducible (Figure 19b). Some of the initial contacts between the reference surface and the plaster develop into plateaus, which do not easily dissolve. These plateaus are at the nuclei of stagnant flow regions which become more prominent as the experiment proceeds, leaving ever-deepening high flow channels between them. Thus the flow channel networks, as observed visually and through numerical simulations, evolves from a homogeneous system to one more (self-) organized and complex.

The fluid permeability across our samples evolves as the plaster substrate is dissolved (Figure 20). A characteristic permeability curve includes four regimes: a short, sharp initial drop, followed by a rapid rise, 


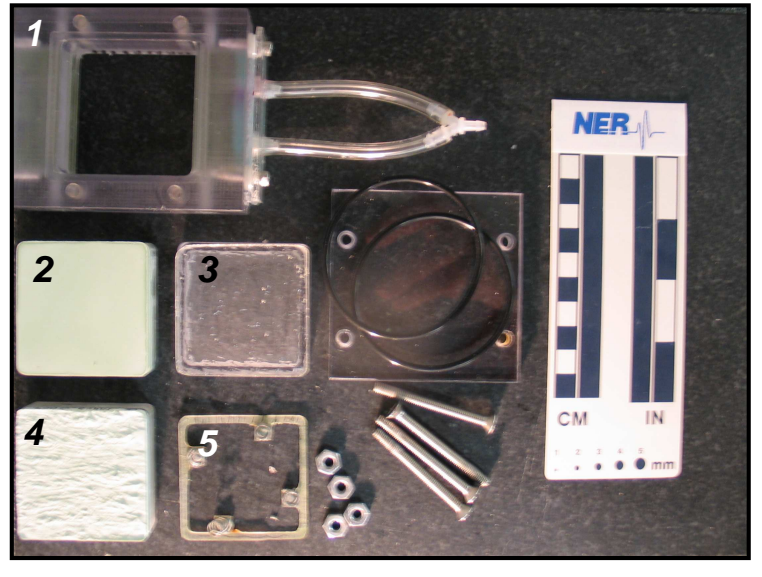

(a)

\section{Laboratory Flow Cell}

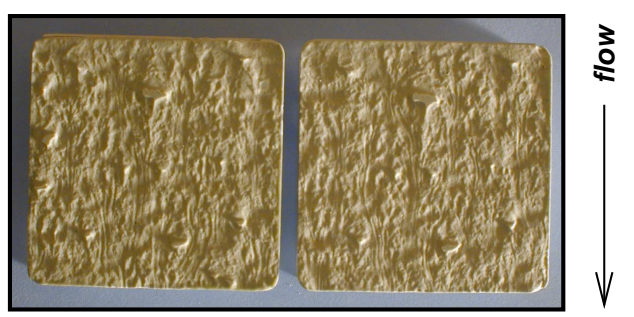

(b) Plaster Samples

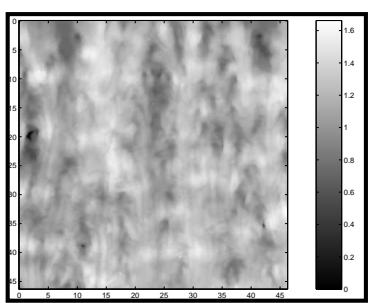

(c) Laser Scan

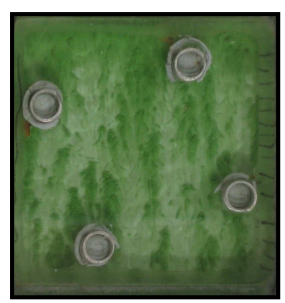

(d) Flow Image

Figure 19: Laboratory set-up for dissolution experiments. (a) Disassembled specimen. The sample holder (1) has integral flow manifolds, which accept the plaster samples (2). A textured cover sheet (3) contacts the plaster surface with a spring mechanism (5) to form a fracture - held together under constant normal stress. After the flow experiment the plaster sample can be removed for detailed study (4). (b) When experimental conditions are the same, the results are reproducible as seen in these two separate samples. (c) The dissolution-induced surface topography is characterized at the end of each experiment by highresolution laser profilometry. (d) The flow geometry is monitored throughout the experiments with digital photography.

then a longer period of pseudo-steady flow, followed by an extended drop. We believe that the first drop is caused by the dissolution of a small amount of material at the contact points. The subsequent increase is likely due to the formation of the stagnant zones around the contact points, precluding further dissolution of these points while flow channels open over the rest of the sample. The plateaus occur during a temporary balance between the burgeoning stagnant zones and the deepening channels. Ultimately, the permeability decreases again as the channels narrow and the stagnant zones grow.

During sampling, we monitor the growth of these stagnant regions through the clear viewing surface, as well as tracking the overall conductivity of the solution. The plaster is dissolved much more quickly at the beginning of the experiment, until the combination of increasing calcium concentration in the pore fluid and decreasing effective surface area brings the dissolution rate to a plateau.

Amongst the dissolution experiments we ran, we noted that shape of the permeability change versus time curve was dependent on the average fluid flow rate across the sample. Figure 20 shows two cases: the blue line represents high-flow $(\sim 3 \mathrm{~L} / \mathrm{hr})$ and the green is a low-flow trial ( $\sim 900 \mathrm{~mL} / \mathrm{hr})$. For each of these flow rates, the four characteristic regimes are present. However, the fast flow sample experiences more abrupt and volatile flow rate changes over the course of the experiment.

Qualitatively, we observed that experiments with lower flow rates formed deep, defined channels that were clearly separated by stagnant regions, whereas those with higher flow rates were prone to sheet-like flow that resulted in few stagnant zones and mimicked the topography of the model fracture. We verified these observations using laser-profilometry to quantify the final topography on low and high-flow samples. Recent results at slower flow rates of $60 \mathrm{~mL} / \mathrm{hr}$ verify this trend.

The final laser profile of a sample may be combined with the profile of the contacting shower glass to create a map of aperture over the entire surface. This enables us to run a finite-difference and lattice 


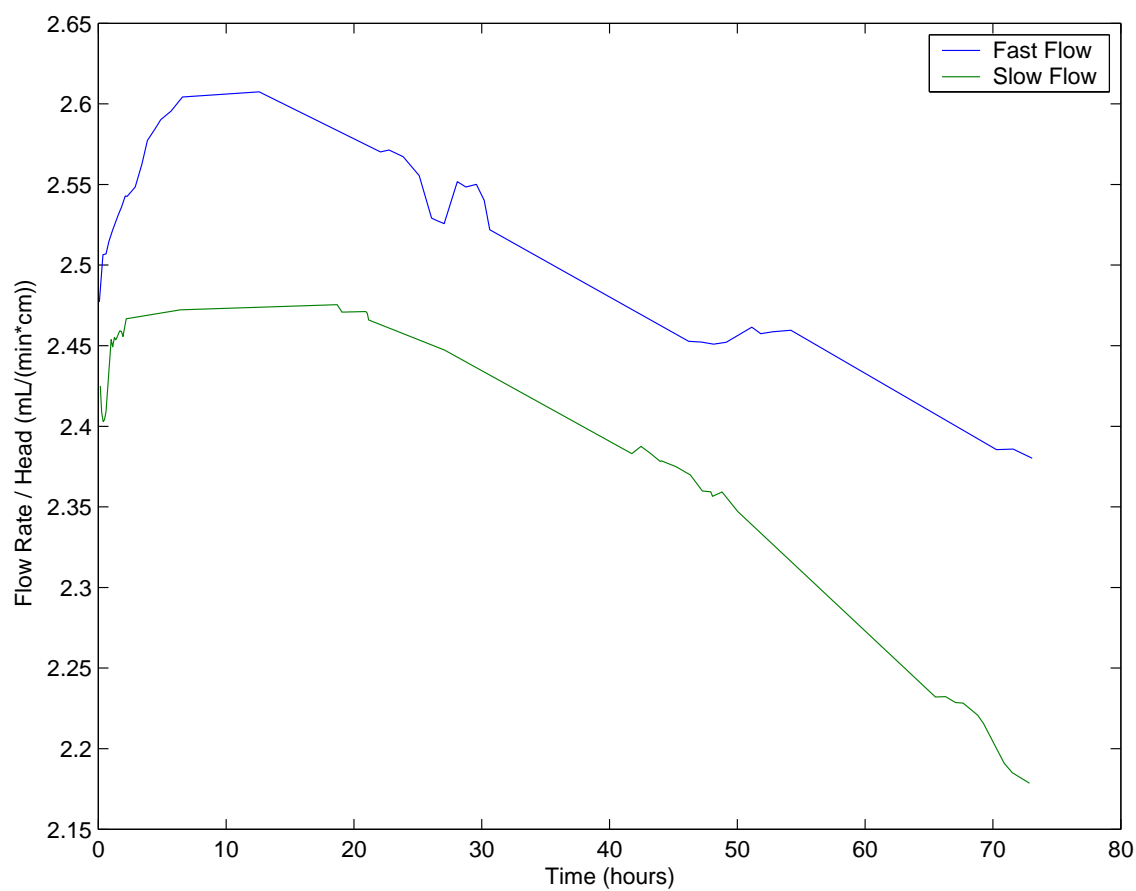

Figure 20: Results of a dissolution-dominated experiment in gypsum plaster. Graph tracking the flow rate (indicating permeability) of a sample over the course of two experiments, one with fast flow, one with slow flow.

Boltzmann simulations of the fluid flow. The results of these simulations verify our suspicions about the different topographies of low and high-flow samples. In the low-flow sample below, relative fluid velocity is much higher in the channels, which provide a direct, non-meandering route from start to finish. This contrasts with the high-flow samples that are less direct and engage more of the fracture surface.

Numerical Modeling In the plaster dissolution experiments, two phenomena recur: 1) plateaus or high areas form opposite projections of the textured glass surface, and 2) stringers - long raised areas of plaster often form in the lee (downstream side) of those plateaus. We have used LB to model the formation of these features, first in idealized geometries that have bumps and depressions like the textured glass; then with an actual texture-glass-type surface as measured by profilometry.

There is a reasonably straightforward explanation for the uneroded projections. The aperture is small beneath the plaster projection and the corresponding textured glass projection; hence flow is likely to be slower between them, and dissolved solute will remain longer near the plaster, keeping the solution near saturation with gypsum. Thus the dissolution rate will be slow, and the topography unaltered. The stringers are a bit more difficult to explain; they require that stable, high-solute concentration plumes exist in the lee of the projection. The existence of such plumes would require a rather high $P e$ number, since the flow would have to be fast enough to limit transverse diffusion.

To test these possible mechanisms, we begin by using idealized geometries for LB calculations. The calculations are scaled so the $D a$ and $P e$ correspond as closely as possible to gypsum transport and dissolution. Figure 21 shows the ideal geometries studied, referred to as A, B and C. The top shows a view down the y-axis for each geometry; the initial flat plaster plate is at the top. The bottom of Figure 21 shows the textures on the glass plates that oppose the plaster, as viewed down the $z$-axis. Geometries B and C have pits added, so that solutes may diffuse into them; the pits can act as a capacitance to smear out the effective 


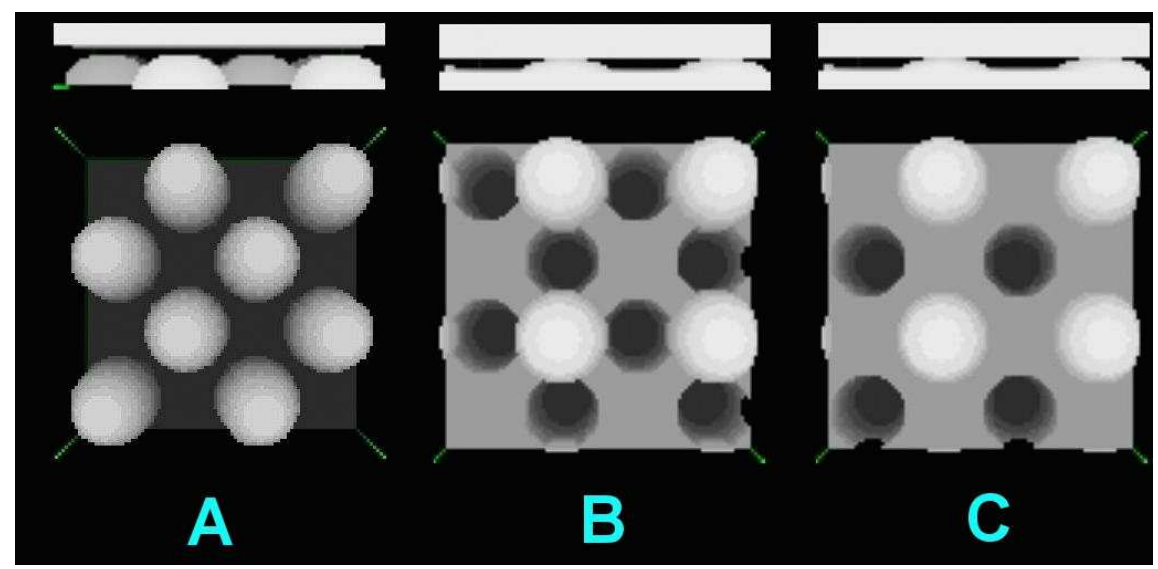

Figure 21: The A, B and C geometries used for the fracture dissolution test. The top three portions of the figure are cross-section views through the "fracture" and the bottom three portions are plan views. These images are 3D perspective projections; the lighter the color, the closer that solid is to the observer.

diffusion coefficient. In $\mathrm{A}$, the top and bottom surface never touch; in $\mathrm{B}$ and $\mathrm{C}$, the two surfaces are in contact at the beginning of the simulation.

The three geometries (A, B and C) all produced substantially the same result: a plateau of "plaster" remained opposite the projections in the textured surfaces, regardless of whether the surfaces were touching or not. Figure 22 shows snapshots of the speed distribution at different positions above the dissolvable plate and at three different time steps, for geometry A. Even though the plaster and textured surfaces were not touching, the diminished flow, in regions of low aperture, suppressed dissolution. Furthermore, "wakes" of solid were left in the lee of the projections, as the simulation progressed.

Next, we attempt to recreate the long stringers in the lee of projections, as seen in Figure 23a. An experimental dissolution cell is simulated, with the caveat that only the central $64 \%$ of the plaster surface is used, and we could reach only $1 / 3$ the $P e$ number of the experiments, in order to keep the calculation time reasonable for this scoping study. Otherwise, the calculation is a fairly realistic representation; the flow into and out of the cell passes over long rectangular prisms, which are meant to simulate the screw flow diffusers used in the real cell. The LB simulation uses a textured surface to oppose the initially flat plaster, taken from an actual glass surface used in the experiments and characterized by laser profilometry.

Figure $23 \mathrm{~b}$ shows the results of the LB simulation. The left side of the figure shows solute concentration, and the right side shows the erosion of plaster surface. The long stringers of high solute concentration, which occur in the lees of projections of the textured surface, correspond to similarly-shaped stringers of high-relief (relatively uneroded) plaster. Thus the LB simulation predicts features similar to those seen in laboratory tests (Figure 23a). We would expect that at higher $P e$, as used in this experiment, the solute stringers would be sharper and narrower (less transverse diffusion), hence would result in narrower and sharper stringers in the plaster surface.

Conclusions We find that when experimental parameters (e.g. initial surface topography, flow rate history, and total experiment time) are duplicated, the topography developed on the laboratory samples is reproducible from one dissolution experiment to the next. The flow channel networks, as observed visually and through numerical simulations, evolves from a homogeneous system to one more (self) organized and complex. The permeability initially drops as the experiment begins and the surfaces settle into place, then gradually rises with several further smaller decreases as some supporting asperities are destroyed. LB methods predict the formation of major dissolution features, such as long "stringers" in the lee of obstacles. 


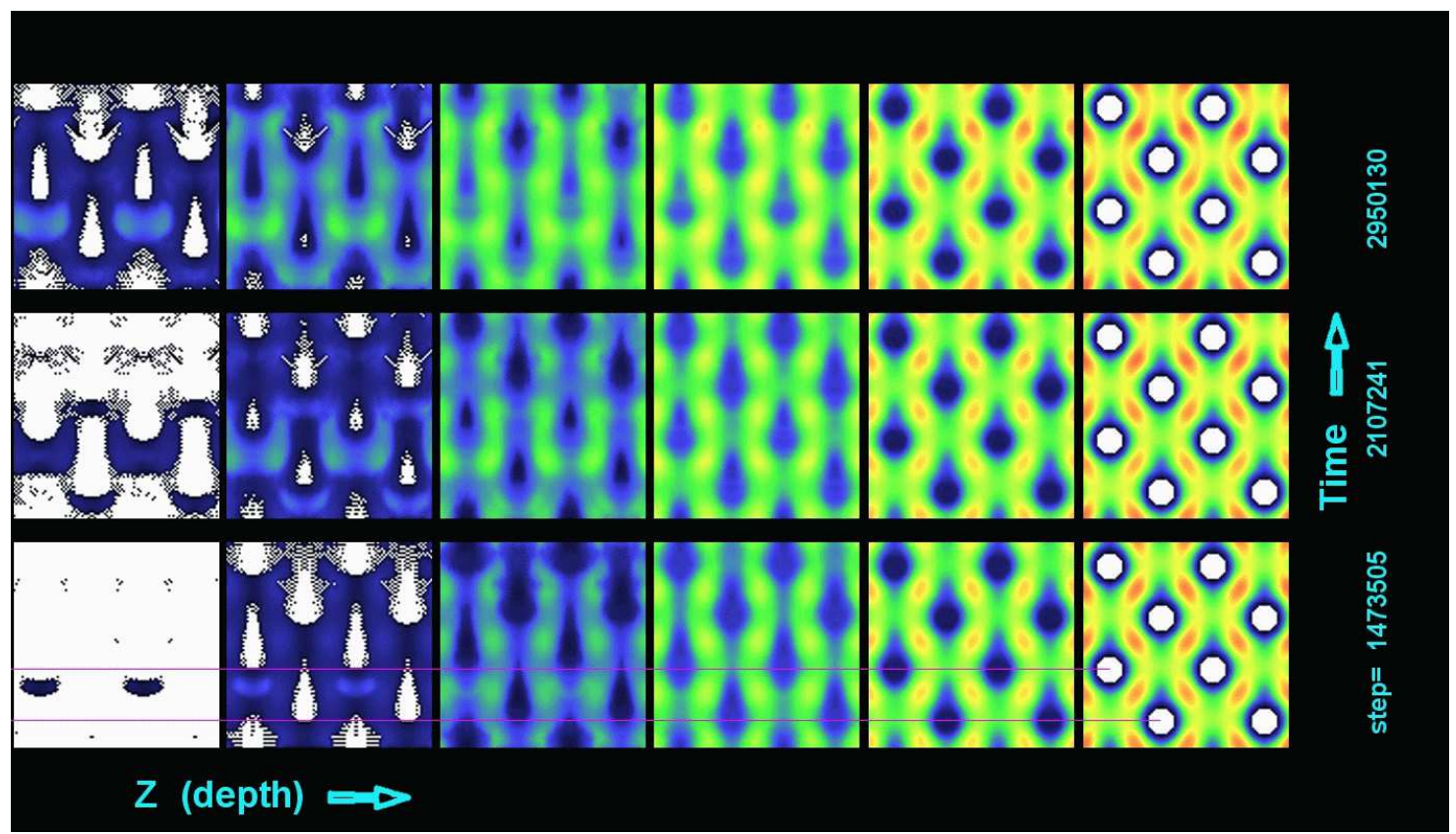

Figure 22: Flow speeds for geometry A, at three different times and 6 positions on the $z$-axis. Solids are white, and flow speed is represented by color, from black for the lowest speed to red for the highest. Flow is from the bottom of the figure to the top. The left of the figure corresponds to the dissolvable plaster plate, and each successive frame to the right is at a lower $z$ position, until the non-dissolvable textured glass is reached at the right hand side of the figure. The magenta vertical lines, in the bottom row, are meant to show the position of the non-dissolvable convex projections (at right) with positions on the dissolvable plate (at left). Plateaus initially form opposite the projections in the non-dissolvable plate, and undissolved tails form in the lee of the plateaus.

\subsection{Observations in Vein Systems (Collaborative Project)}

Abstract We supported a study of a natural fracture system performed at the University of Utah under the direction of Professor Ron Bruhn. Through digital photography and image analysis we have examined natural vein fillings and geometry in drill core from the Awibenkok Geothermal Field. These data will be used as a guide for our physical modeling experimental design and data analysis. However, so far, since the conclusions are derived from veins - which are dominated by precipitation - different interpretations of the permeability history result as compared to the dissolution experiments just described.

Methods We selected core intervals for direct inspection based on vein mineral type and geometry. Geometrical properties of interest included (a) single veins, (b) vein intersections, and (c) veins containing alteration halos or selvages. Several fault-controlled veins were identified and also selected for sampling in addition to the more commonly occurring extensional veins. Digital images of the veins were collected using a flat-bed scanner. Also, a digital camera under ultraviolet light was used to record details of vein geometry revealed by fluorescence of calcium carbonate minerals. Thin sections of some specimens were prepared. Rubber casts were also made of several vein walls to prepare samples for measuring wall roughness. We developed a procedure to partially automate measurements of vein thickness, area, and roughness directly from the digital images (Figure 24). 


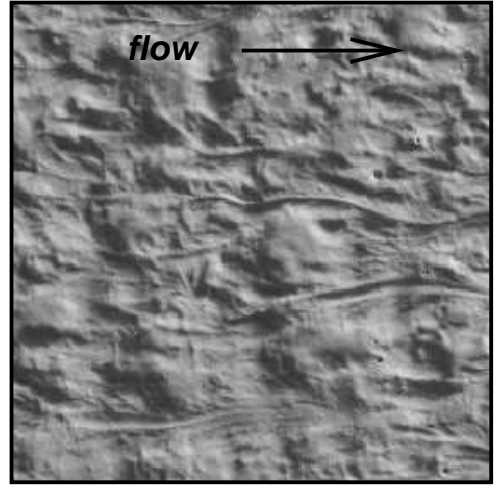

(a) Plaster Sample

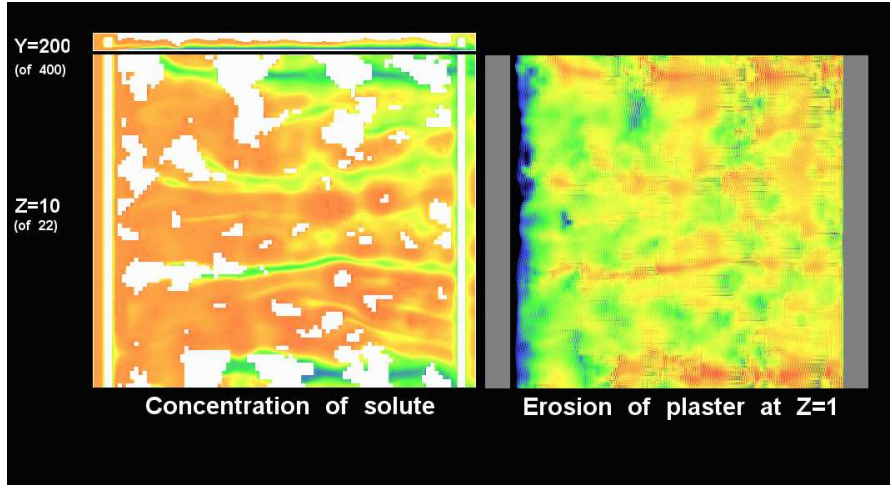

(b) Lattice-Boltzmann Simulation

Figure 23: (a) Section from an initially flat plaster surface after a dissolution experiment; flow from left to right. Note the long stringers in the lee of projections; the projections themselves typically correspond to projections in the opposing textured glass. (b) Simulation of chemical erosion in plaster flow cell. The left side gives concentration at two cuts through the automaton; an $\mathrm{x}-\mathrm{z}$ cut (top, view down $\mathrm{y}$ axis) and a $\mathrm{x}-\mathrm{y}$ cut (bottom, view down $\mathrm{z}$ axis); solids are white, and concentration varies from red (lowest) to blue (highest). On the right is shown the erosion depth on the plaster surface, from red (lowest, to blue and black (highest erosion). Long stringers similar to those in (a) are evident.

Conclusions We found that multi-phase mineralization due to chemical dissolution and re-precipitation as well as mechanical fracturing was a common feature in many of the veins and that it had a significantly affected interpretations of vein tortuosity and permeability history. We used our microand macro-scale observations to construct four hypothetical permeability models (e.g., Figure 25). In each model, permeability changes, and in most cases fluctuates, differently over time as the tortuosity and aperture of veins are affected by the precipitation, dissolution, and re-precipitation of minerals, and also by mechanical fracturing. In all four cases we interpret a first-phase mineral dissolution stage where permeability gradually declines as the vein is blocked by inward growing minerals. Thereafter, permeability may briefly increase with the onset of internal fracturing within the vein or by a phase of mineral dissolution opening up new pathways for fluid flow. Eventually we infer that permeability will decline again as second stage minerals are deposited in the fluid flow pathways.

\subsection{Precipitation in Fractures (Future Directions)}

Abstract We performed new LB calculations of precipitation in fractures and preliminary laboratory experiments to guide our thinking for future work on precipitation within fractures. We limited these studies to fully saturated fracture systems. The LB modeling work was performed by our collaborator H.W. Stockman of Sandia National Laboratories and the experimental work was performed at New England Research.

The calculations first deal with of growth along the large direction of the fracture, parallel to the fracture plane. This might be the dominant type of growth if the precipitate had little or no chemical affinity for the fracture walls; for example, if the walls were made out of tuff, and the precipitates were a very different material (such as hydrous uranium oxide or manganese oxide. In this case, growth will travel out from a chance seed with greater chemical affinity for the precipitate - perhaps by either a chance nucleation directly from solution or precipitation onto a surface with a similar surface tension. Eventually, we intend to experimentally model such growth with special cells, using gypsum and borax growing between Plastic plates. Growth of precipitates from this mechanism might also occur if there were large compositional gradients along the plane of the fracture, as might accompany mixing of fingers of very different solutions. 


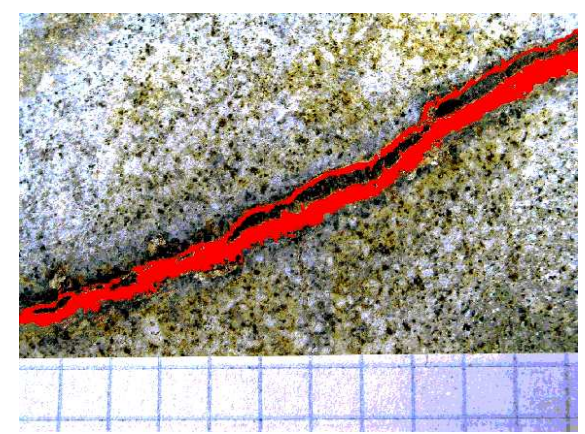

(a)

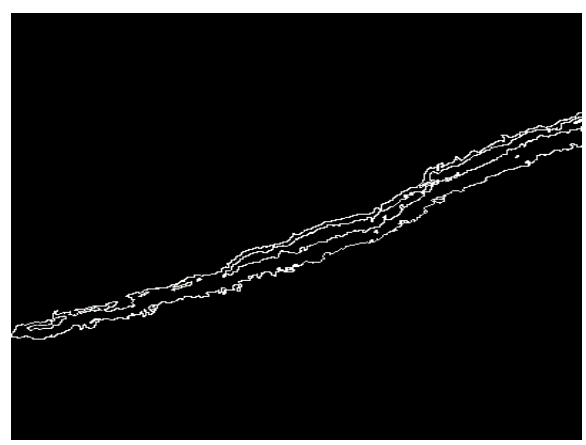

(b)

Figure 24: Example images of vein geometry in Awibenkok core. (a) Awiebenkok core sample from a depth of 1186 feet with calcite vein digitized as a red ROI (Region of Interest). Notice the complex geometry of the vein margins, the variability in vein thickness, and the altered remnants of wall rock incorporated into the vein. Each grid spacing is $1 \mathrm{~mm}$. (b) Enhanced image of vein margins for the calcite vein on the left. This type of image is prepared as part of further analysis of vein geometry.

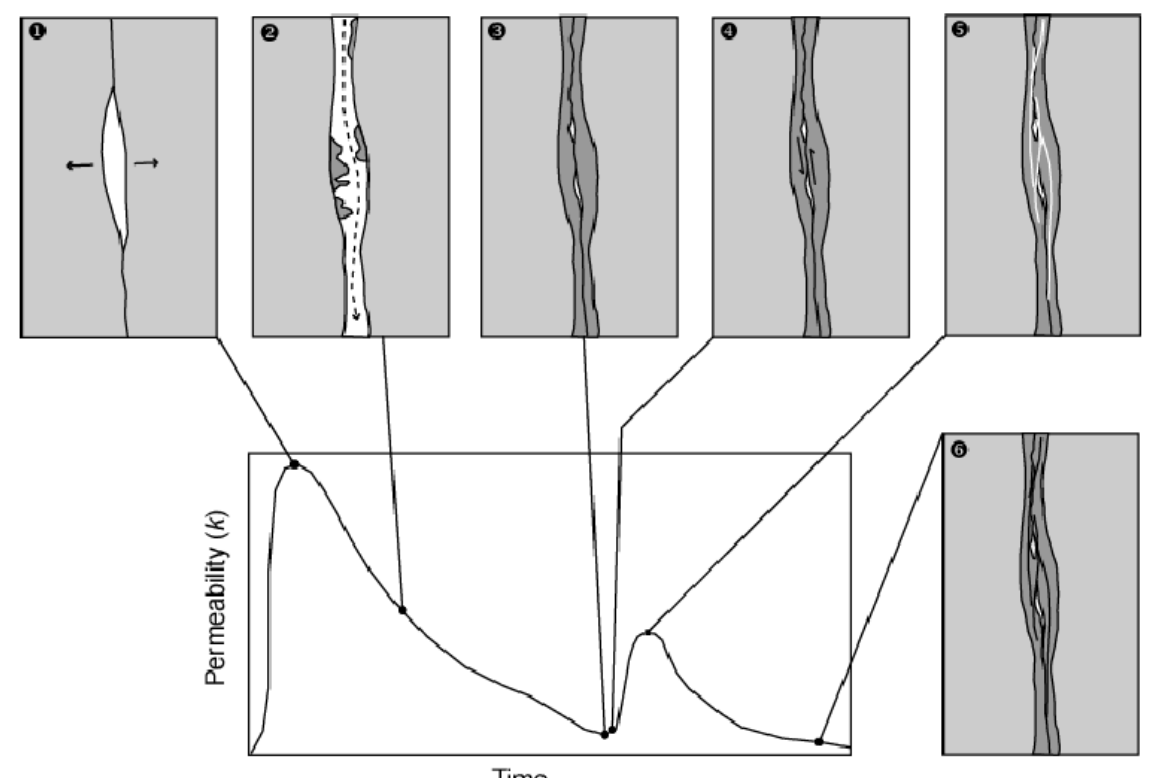

1) Fracture opens by tensile fracturing. Permeability is high and fluid flows enters void space

2) Minerals are precipitated in the vein, growing outward from the vein walls into the middle of the vein obstructing flow and increasing tortuosity

3) The vein closes and permeability decreases markedly as minerals continue to grow inward.

4) Renewed fracturing disrupts mineral grains and permeability increases once again.

5) Growing fractures connect together to form a new network, permeability rises rapidly and fluid flow increases.

6) Minerals precipitate and once again reduce permeability.

Figure 25: One of several permeability evolution models suggested by inspection of vein geometries in Awiebenkok core samples. 
For example, a sulfate-rich finger, and a calcium-rich finger might intermix, precipitating gypsum, or a finger rich in basic uranium carbonate solution might mix with lower-pH fluids containing little carbonate, as the uranium-rich finger cascades down through fractures in the tuff, from a waste-package source.

Next we deal with growth onto a substrate of like material. An example might be the growth of gypsum on a large planar surface of plaster, as we have used previously in our dissolution experiments. In this situation, the solution is kept marginally over-saturated, and precipitates initially form all over the large "plaster" surface, reducing the permeabilty. Then the precipitates "ripen," rearranging to reduce surface tension, so that flow channels open up and one again increase the permeability.

Finally, we present new experimental results, pertaining to growth on a large plaster surface. The aim of these new tests is to show that experimentally one can choose conditions for precipitation, such that: 1) the precipitation occurs simultaneously with dissolution; 2) the precipitates form only on the pre-existing seeds (in this case, a plaster (gypsum) surface), and not in the tubing, mixing chamber, or Plastic viewing wall of the cell; 3) the flow speed through the cell is sufficiently short to avoid spontaneous nucleation, but long enough to achieve seeded precipitation, and still provide a relevant range of $P e$.

Lattice Boltzmann simulations To begin, we ask a fundamental question: what effect does flow have on the speed of dendrite growth? Figure 26 shows four LB simulations designed to answer this question, at different steps in the simulation $(200 \mathrm{k}, 300 \mathrm{k}$ and $400 \mathrm{k}$ from left to right). At top are concentration plots from a simulation with no flow; solids are white, and the solute concentration ranges from red (lowest) on the left margin, to blue (highest) on the right. The right boundary is kept at a constant concentration, and the initial solute concentration is super-saturated. Solid walls are placed on the left and right, and the left wall is a "seed" capable of precipitating the solid. The very top simulation has no flow and no solid boundaries except those on the left and right; effectively, the simulation is infinite in the $z$-direction. The second row also has no flow, but this time the $z$-direction is constrained by solid walls, producing a Hele-Shaw cell. In these first two simulations, dendritic growth is minimal in the time of the runs. But in the bottom two rows, when flow is added, dendrites readily grow from the sidewalls, pointing into the flow.

The cause of much faster dendrite growth under flow is fairly simple: the flow past the tip of the dendrite keeps the concentration gradient steep, by constantly replenishing with new solution; thus the tip sees a high flux of solute. Since reaction is, by definition, fast compared to diffusion, the rate of growth is almost entirely determined by the diffusion gradient. Meanwhile, the fast-growing dendrite leaves a shadow of solute depletion in its lee, effectively starving-out the competition, preventing nearby dendrites from growing.

Now that enhancement from flow is obvious, what is the effect of restricting the flow between two plates in a Hele-Shaw cell? One might expect that the effect of Taylor-Aris dispersion would be to smear concentration in any flow that has an $x$-direction component, lessening the dendrite growth. But as the bottom of Figure 26 shows, a dendrite grows into the flow in the Hele-Shaw cell, perhaps at an even more acute angle, and at least as quickly.

We have seen that LB models predict that a flow field greatly enhances the growth of dendrites, and the enhancement persists when the growth is confined in a smooth-walled "fracture" (Hele-Shaw cell), with the dendrites growing into the flow. But what if the flow field is complicated by roughness?

Figure 27 shows the results of calculations, in which one of the smooth walls is replaced with a rough wall, which produces a complicated flow field between the plates. Six steps in the calculation are shown in the figure, with time progressing from left to right. During the simulation a whitish dendrite grows on from the left side, and nearly chokes off the flow by step 500k. However, the dendrite did not simply grow "into" the bulk fluid flow direction as did the dendrites in Figure 26; in fact, it is pointing somewhat backwards.

Careful examination of Figure 27 helps explain why the dendrite picked this peculiar growth pattern. At the top of the figure, the concentrations of solute are shown, from blue (highest concentration) to red (lowest). As the one dendrite grows and becomes dominant, it leaves solute-depleted fluid in its wake, 

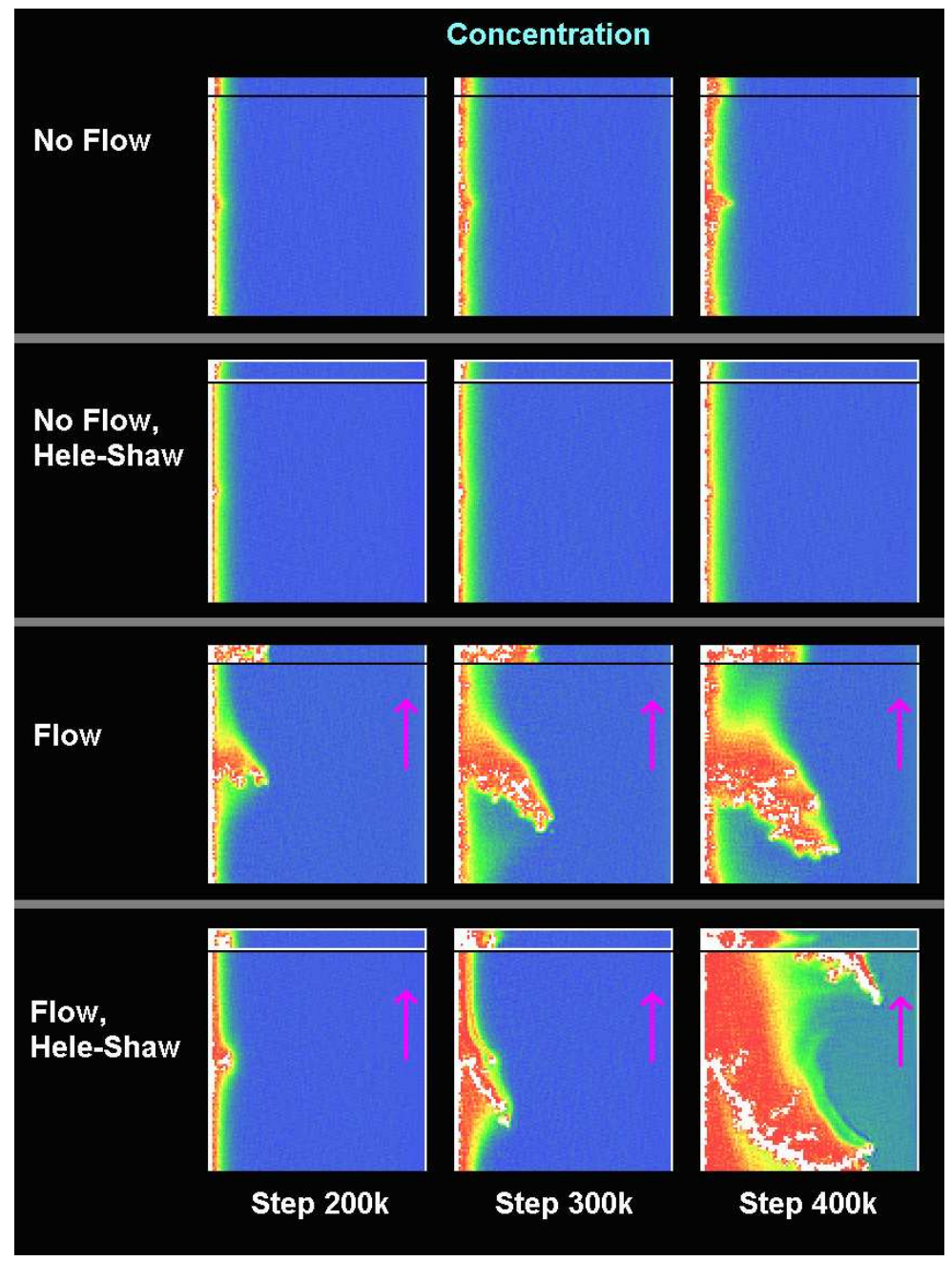

Figure 26: Dendrite growth in 4 separate conditions (see text). Solids are white; concentration of solute grades from red (lowest) to blue (highest). The red arrows show the direction of flow in the two bottom simulations.

effectively choking off the growth of other dendrites. It is clear that the one finger has grown toward the fastest flow, wherever it was available; note there is a red region at the dendrite tip, in almost every frame. The flow field is complicated by the roughness of the bottom surface, and so is the path of the dendrite complicated, in seeking the fastest flow, where the diffusional gradient will correspondingly be the smallest.

Next consider precipitation that occurs by the convergence of two fluid streams, such that the mixture of the two streams is saturated with a solid phase, but the individual streams are not saturated. An example would be the convergence of $\mathrm{CaCl}_{2}$ in water, with a stream of $\mathrm{Na}_{2} \mathrm{SO}_{4}$ in water; the admixture of the two might be supersaturated with respect to gypsum. Figure 28 shows a calculation for such a system; a $\mathrm{CaCl}_{2}$ stream flows up from the bottom of the left, and a $\mathrm{Na}_{2} \mathrm{SO}_{4}$ stream flows up on the right. The top row of Figure 28 shows successive steps in a Hele-Shaw cell, with smooth top and bottom walls, which act as substrates for the precipitation. $\mathrm{CaSO}_{4}$ does precipitate at the juncture of the two flows; but the precipitate quickly chokes off the exchange of solute between the two sides of the flow, so precipitation effectively stops by step $314 \mathrm{k}$. The bottom row shows the case where the top wall is textured, so a non-uniform flow field is imposed, and causes a greater mixing of the streams. More precipitate forms at the junction, probably due to the initially greater mixing, but once again the exchange is choked off. While this example may seem 


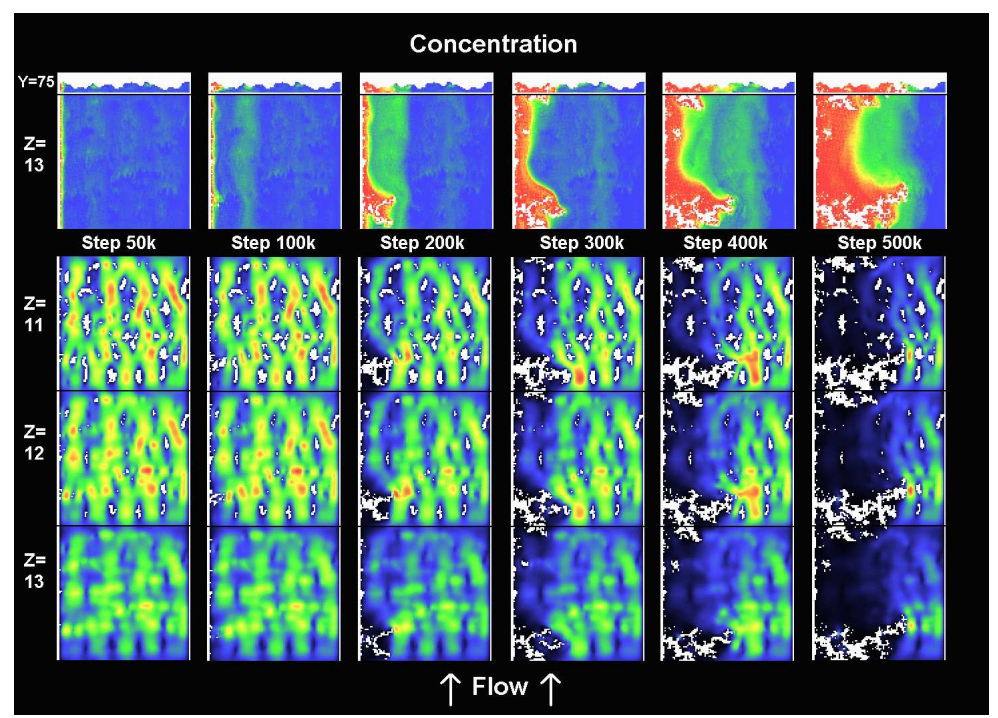

Figure 27: Dendrite growth when the top of the cell is rough (see text). The top row shows the concentration of solute; the bottom 3 rows show flow speed at successive $\mathrm{Z}$ levels. The dendrite grows toward the fastest flow, which corresponds with the steepest concentration gradient.

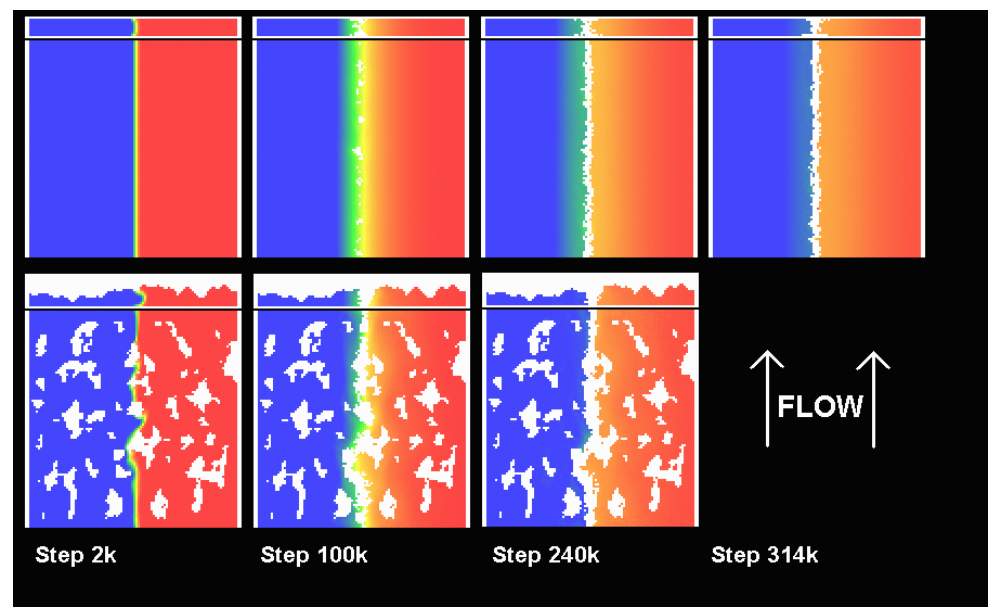

Figure 28: Growth of solids (white) when Ca-rich stream (blue) and a sulfate-rich stream (red) mix (see text). The top is a flat-walled Hele-Shaw cell; for the bottom, the upper surface is textured.

naive, it succinctly illustrates a fault built into many bulk treatments of precipitation: two fingers of different composition are presumed to meet, mix, and produce a precipitate consistent with the net composition. But on the microscopic scale, mixing may be greatly limited by occlusion from the first precipitates.

Precipitation and Dissolution in the Same Fracture The next example is closely related to our previous experiments involving dissolution only. The geometry is illustrated schematically in Figure 29. This time the bulk flow is from left to right. The automaton has 100 nodes in the $x$ - and $y$-directions, and 20 in the $z$-direction. At $z=0$, a "plaster" surface is placed, and this surface is capable of precipitating gypsum. The plaster surface is opposed by a rough textured surface, which creates the initial variations in the flow field. The textured surface is analogous to the textured "shower glass" used in our experiments, but here the topography of the surface is exaggerated for illustration purposes. 


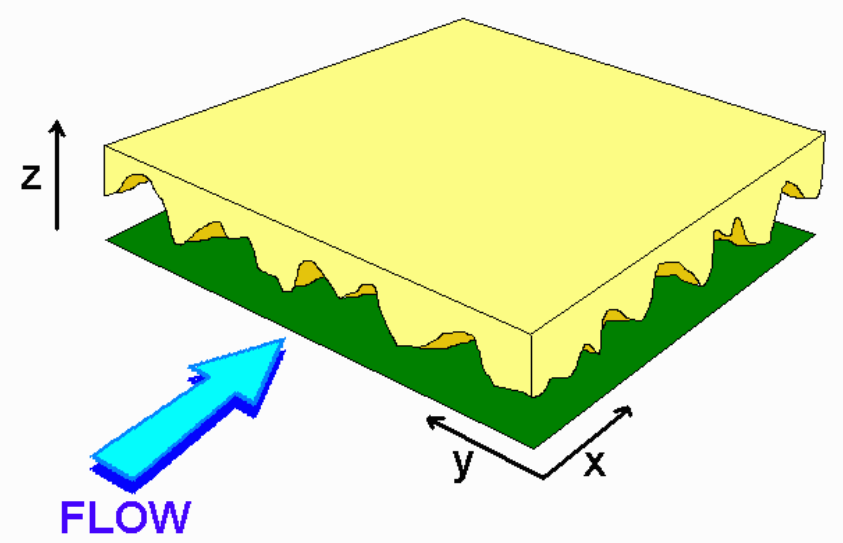

Figure 29: Axis orientation for geometry in Figure 30.

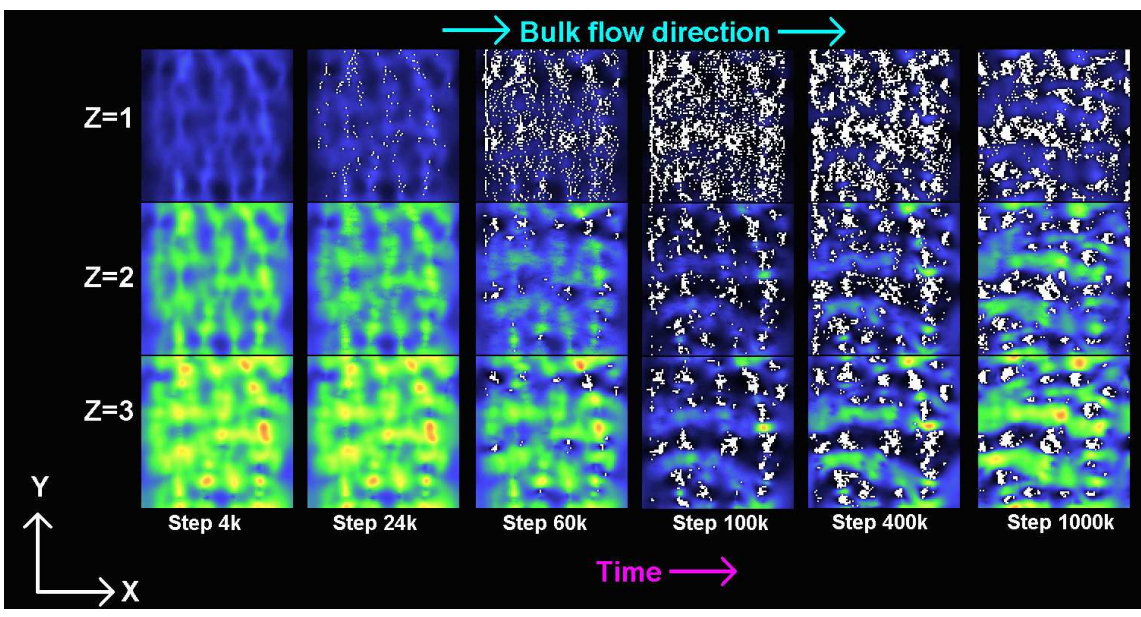

Figure 30: Flow speed plots for successive positions above the plaster surface (see Figure 29 for orientation). The solids are white; flow speed is plotted, from black (no flow) to red (highest speed flow). Initially precipitates form all over the $z=1$ surface, slowing flow; but as time progresses, the precipitates ripen to form more coherent masses, and flow channels develop between the masses, increasing the permeability.

Figure 30 details the evolution of precipitates at three (successively higher) z levels just above the "plaster" plane. Solids are white, and the other colors represent flow speed, from black (no flow) to red (highest flow speed). The figure shows six time steps, arranged from left to right. At the earliest time step on the left (4k), no precipitates have yet formed. At the next step (24k), precipitates have formed on the lowest fluid layer $(\mathrm{z}=1)$, approximately where the flow was previously fastest. But, the first precipitates immediately alter the flow field, so subsequent precipitates are difficult to predict. Note the situation here is inherently different from what was illustrated in Figures 26 and 27, because the entire $\mathrm{z}=0$ surface can induce nucleation. Thus growing clumps of precipitates quickly interfere with each other, but there is no bias toward letting one finger dominate and control growth parallel to the z plane. From step 100k to step 1000k, there is not a great deal of change in the amount of precipitates; but the precipitates gradually consolidate to minimize surface area, and eventually allow reestablishment of flow channels through the automaton; so the effective permeability first decreases, then gradually climbs to a near steady-state by step 1000k, as precipitates consolidate, and provide less blockage to the flow field. 
Laboratory Demonstration Here we demonstrate, that with only slight modification to our experimental setup, we can perform experiments dominated by precipitation.

We prepared stock solutions of $0.06 \mathrm{M} \mathrm{Ca}^{++}$and $0.06 \mathrm{M} \mathrm{SO}_{4}^{=}$, from $\mathrm{CaCl}_{2}$ and $\mathrm{K}_{2} \mathrm{SO}_{4}$ reagents. The solutions were added $1: 1$ in a mixing chamber, producing a solution that was $0.03 \mathrm{M}$ in calcium and 0.03 $\mathrm{M}$ in sulfate - at least 2 times over-saturated with respect to gypsum (the ionic strength of the solution was sufficient to raise gypsum solubility above the infinite dilution value).

The experimental flow manifold was loaded with a pristine wafer of plaster. Eighteen rubber cylinders 3 $\mathrm{mm}$ in diameter were inserted between a smooth transparent glass plate and the sample surface, so as to form an array of geometrically arranged obstructions within the simulated fracture (Figure 31a). The aperture of this "fracture" is estimated to be uniform at about $0.5-1 \mathrm{~mm}$. The sample assembly was presaturated with distilled water for one hour and a 0.06 molar solution of $\mathrm{Ca}^{++}$and $\mathrm{SO}_{4}^{=}$, mixed in a small mixing chamber ahead of the flow cell intake, was injected at a constant flow rate of $60 \mathrm{ml} / \mathrm{h}$ for 114 hours. Since the induction time of gypsum for unseeded growth is nearly 0.5 hour, there was no clogging of the apparatus upstream from the plaster.

After disassembly of the specimen, low angle lighting revealed that both dissolution and precipitation of gypsum had occurred along the sample surface (Figure 31b). Areas below the cylindrical rubber obstructions were unaffected by the solution-precipitation process and appear either as topographic highs or lows. Near the intake manifold, strong dissolution results in the formation of well defined channels carved out of the plaster starting material whereas precipitation is dominant as the flow progresses leeward into the sample. This is marked by a broadening of the channels and the formation of plateaus around the cylindrical obstructions. Under magnification these plateaus can be seen to be covered with small euhedral gypsum crystals with their long axis oriented sub-perpendicular to the flow direction. This is in marked contrast to experiments under pure dissolution conditions, where all points (except the plateaus described earlier) are topographic lows (Figure 31c).

We speculate two possible causes for why dissolution occurred at all in these experiments. First there was possible incomplete mixing of the Ca-rich and sulfate-rich streams before entering the cell, so that local fluid streams were not saturated with gypsum. The Hele-Shaw cell would then mix the streams by the Taylor-Aris effect, as flow progressed downstream. Second, it is possible that seeded precipitation of gypsum has a small but finite induction time, especially in the presence of minor impurities. Previous (literature-published) tests found no induction time, but had limited resolution and could not determine induction times of a few minutes. In our experiment, the average linear flow rate was $\approx 0.04 \mathrm{~cm} / \mathrm{s}$, and faster channels with several times that flow rate formed at the leading edge. Thus an induction time of only seconds could make a significant difference in our experiments.

Conclusions The reproducibility of our previous experimental results on dissolution in fractures and our understanding of these results through numerical models leads us to consider the effects of precipitation on fracture flow. Our previous studies of the effects of Pe on fracture mixing [Stockman et al., 2001], and the recent work by Detwiler et al. [2003], lead us to expect strong effects of Peclet number $(\mathrm{Pe})$ and the Damkohler number $(D a)$ on patterns of dissolution and precipitation. The new scoping studies discussed here, including both numerical modeling and experimentation, allow us to hypothesize that:

- Precipitation and dissolution within fractures are both markedly affected by flow rate.

- The geometry of precipitation is markedly affected by the affinity of the fracture walls for the precipitating solid. When there is little affinity for the fracture walls, dendritic growth can proceed out along the plane of the fracture, seeking conditions (typically higher flow rates) that will provide steep concentration gradients. 

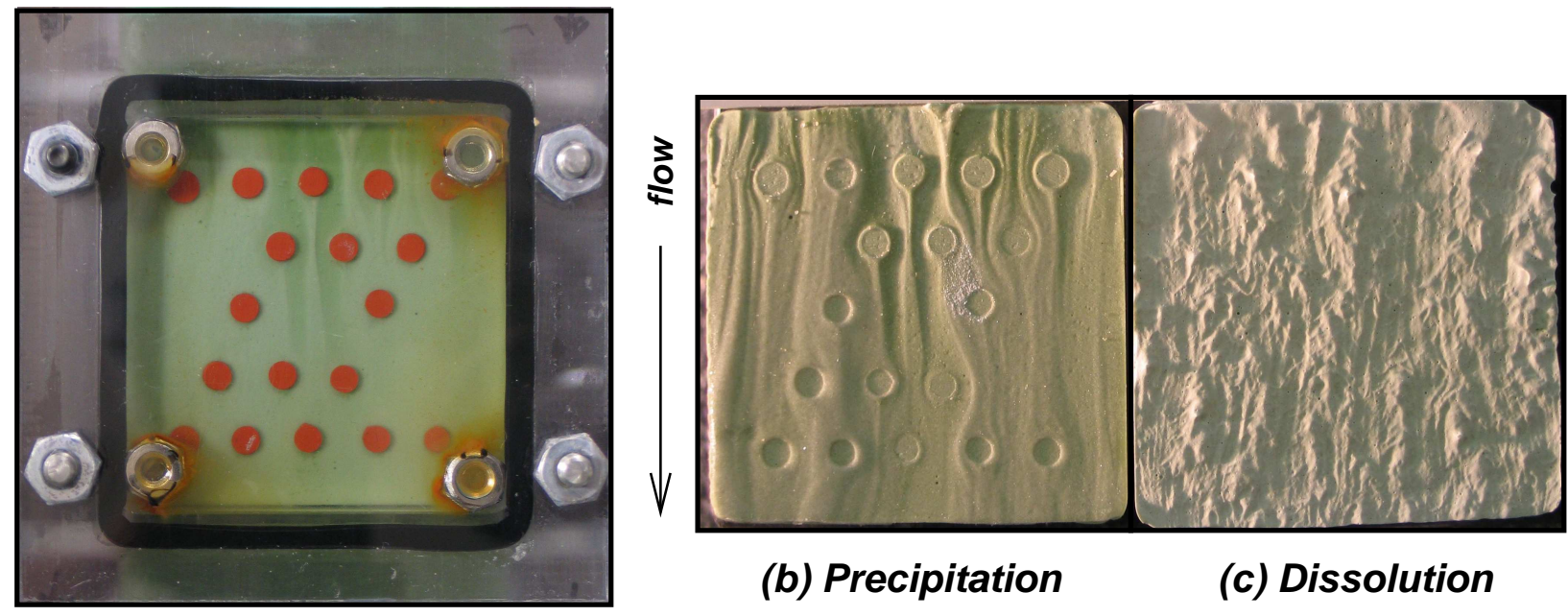

(b) Precipitation

(c) Dissolution

(a) Precipitation Experiment

Figure 31: Experiment involving mixed dissolution and precipitation. (a) The sample consists of a plaster wafer (green) and a smooth glass plate separated by $3 \mathrm{~mm}$ diameter rubber discs (red) to form a Hele-Shaw cell with an aperture of 0.5 to $1.0 \mathrm{~mm}$. The flow is from top to bottom at the constant rate of $60 \mathrm{ml} / \mathrm{h}$. During the experiment we see evidence of channeling parallel to the flow direction. (b) Photograph of plaster sample after removal from the flow manifold. Traces of the rubber discs appear either as topographic highs or lows corresponding to areas of surrounding dissolution or precipitation respectively. Strong channeling is developed near the intake area whereas plateaus are dominant with wider, shallower channels developed leeward. (c) In contrast, here is shown and example of typical dissolution-only experiment run under the same conditions as the previous experiment but with distilled water only and higher flow rates $(1.8 \mathrm{~L} / \mathrm{h})$. Numerous channels are present in this case, but less developed (shallower) and sinusoidal though continuous.

- As dissolution and precipitation proceed, the resulting flow pathways and the bulk permeability will evolve and self-organize, perhaps in response to "ripening" from minimization of surface tension, and small fluctuations in the local concentration of solutes.

\section{Proposed Future Work}

To test these hypotheses and to advance our understanding toward a predictive capability, we suggest that this project could be extended to further physically model and analyze several configurations of flow and transport of chemically active fluids through channels in single fractures, through fracture intersections, and simple fracture networks. This would be an integrated program utilizing quantitative and visual observations of flow and chemical precipitation within synthetic, rough-walled fractures, replicas of real rough-walled fractures, numerical modeling via LB methods, and quantitative observations from fractures and veins in outcrops and in drill core.

To fully understand this problem we would first limit our work to fully saturated fracture systems and vary the physical and chemical properties within this restriction.

In order to accomplish this we would study the factors controlling the evolution of fluid fracture system permeability in the presence of reactive fluids. We would look at three distinct scales of fracture flow and elucidate the processes which predominate for each: (1) single fractures - dissolution and precipitation as affected by surface roughness and as affected by the interaction with pore fluid within the porous matrix rock, (2) dissolution and precipitation as heterogeneous fluids mix within single fractures and fracture 
intersections, and (3) dissolution and precipitation in correlated high flow pathways within heterogeneous fracture networks. All three scales would first be studied under fully saturated conditions with miscible, yet heterogeneous fluids. Furthermore to explore applications of these issues to oil and gas production and nearsurface environmental applications in the vadose zone we would perform scoping laboratory experiments to study the unsaturated aspects of the interaction of the fracture and fluids within the porous matrix for some idealized air/water and oil/water saturation conditions. The idealized laboratory and numerical studies would be guided by observations on natural fractures and veins in the field and in drill core.

Basic sub-topics for study would include the study and quantification of:

1. The development of statistics for distinguishing the geometrical changes that take place in response to flow, and correlation of the statistics with $P e, D a$ and the statistical properties of the initial geometry.

2. The time evolution of system permeability and its relation to the underlying flow channel geometry.

3. The heterogeneous spatial distribution of precipitates - as a means, for example to help understand geostatistics of metal ores. We have seen, for example, that dendrite growth across a fracture can essentially stop flow - and further precipitation - yet fill the channel only sparsely with solid material. Dendritic ores do develop in nature, and are sporadic and currently unpredictable. Yet the density of dendrite filling must be controlled by the $P e, D a$, and initial geometry.

We emphasize the development of statistical measures in the data analysis. Our experience shows us that it is very difficult to produce a simulation that exactly models experimental flow; small variations in the initial uniformity of the flow field, the density of the plaster, etc., can have a strong influence of the development of specific dissolution patterns. Yet the average size and spacing of dissolution patterns is much easier to reproduce in models. With precipitation, we can only expect the situation will worsen, given the chance nature of dendrite growth. Hence we must develop the means to extract meaningful statistics from our data, that will be reproducible, even when the exact flow patterns are not.

\section{Publications Resulting From This Project}

Stockman, H.W., A 3D Lattice Boltzmann Code for Modeling Flow and Multi-Component Dispersion, Sandia Laboratories Technical Report, SAND99-0162, February, 1999.

Tripp, A.C., R.L. Bruhn, and S.R. Brown, Inductive detection of fractures in geothermal systems: Petrophysical aspects and geophysical implications, in Proc. Twenty-fourth Workshop on Geothermal Reservoir Engineering, Stanford University, Stanford, California, January 26-28, 1999.

Johnson, J.P., and S.R. Brown, Solute transport in actual fracture intersections compared to parallel plate models, Eos Trans. AGU, Fall Meet. Suppl., Abstract H52C-04, 2000.

Renshaw, C.E., Dadakis, J.S., and S.R. Brown, Measuring fracture apertures: A comparison of methods, Geophys. Res. Lett., 27, 289-292, 2000.

Johnson, J.P., and S.R. Brown, Mixing variability in natural fracture intersections. Geophys. Res. Lett., 28, 4303-4306, 2001.

Johnson, J.P., S.R. Brown, and H.W. Stockman, Surface roughness effects on solute dilution and dispersion in idealized fracture networks, Eos Trans. AGU, Fall Meet. Suppl., Abstract H61B-12, 2001.

Stockman H.W., J.P. Johnson, and S.R. Brown, Mixing at fracture intersections: influence of channel geometry and the Reynolds and Peclet numbers, Geophys. Res. Lett. 28, 4299-4302, 2001.

Ebel, K.A., and S.R. Brown, Evolution of fracture permeability, Eos Trans. AGU, 84(46), Fall Meet. Suppl., Abstract H42F-1134, 2003.

Khatwa, A., R.L. Bruhn, and S.R. Brown, Geometric analysis of vein fracture networks from the Awibengkok core, Indonesia, Eos Trans. AGU, 84(46), Fall Meet. Suppl., Abstract H42F-1137, 2003. 
Renshaw, C.E., T.A. Myse, and S.R. Brown, Role of heterogeneity in elastic properties and layer thickness in the jointing of layered sedimentary rocks, Geophys. Res. Lett., 30, 2295, 2003.

Johnson, J.P., S.R. Brown, and H.W. Stockman, Fluid flow and mixing in rough-walled fracture intersections, manuscript under revision, J. Geophys. Res., 2006.

Khatwa, A., R.L. Bruhn, and S.R. Brown, Geometric characterization of vein wall structure, manuscript in preparation, 2006.

Johnson, J.P., and S.R. Brown, Surface roughness effects on solute dilution and dispersion in idealized fracture networks, manuscript in preparation, 2006.

Brown, S.R., and M.L. Smith, A transient-flow syringe air permeameter, manuscript in preparation, 2006.

Caprihan, A., and S.R. Brown, MRI studies of fluid flow in rock fractures, manuscript in preparation, 2006. 


\section{Commercial Products Developed}

The "TinyPerm" syringe permeameter was developed further with additional government and private funding. For ease of use, it now uses a vacuum flow system and an internal micro-controller implementing the required signal processing algorithm. It is now a commercial product available for sale from New England Research (see below and http://www.ner.com). A patent application was not submitted for this device, since market research suggested that the cost/benefit for patent application fees was too high compared to potential sales.

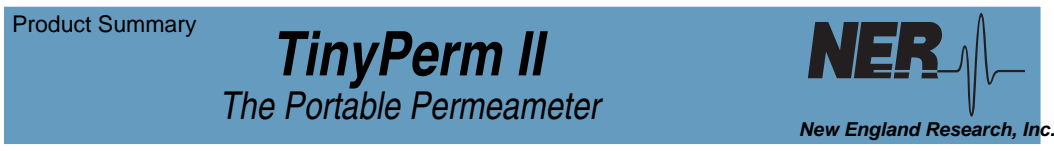

NER's TinyPerm Ilis a portable hand-held air permeameter used for measurement of rock matrix permeability or effective fracture apertures on outcrops and at the core scale.

\section{Operation:}

The operator presses a rubber nozzle against the specimen and withdraws air from it with a single stroke of a syringe. As air is pulled from the sample, a micro-controller unit simultaneously monitors the syringe volume and the transient vacuum pulse created at the sample surface. Using signal processing algorithms the micro-controller computes the response function of the sample/instrument system. Key characteristics of this response are displayed on the liquid crystal display (LCD). Theory shows how the response function is related to permeability and either matrix permeability or effective fracture flow aperture can be determined from the calibration charts and tables provided.

For intact rock, the permeability measurement range is from approximately 10 millidarcys to 10 darcys. Similarly, fracture aperture from approximately 10 microns to 2 millimeters can be determined.

\section{Features:}

Can be operated by one person. Easily carried in a small backpack. Physical size: syringe unit $-38 \mathrm{~cm} \times 12.5 \mathrm{~cm} \times 5 \mathrm{~cm}$ microcontroller unit $-16.5 \mathrm{~cm} \times 11.5 \mathrm{~cm} \times 5 \mathrm{~cm}$ interface cable -3 meters

Total weight (excluding carrying case)t. $2 \mathrm{~kg}$

Microcontroller unitMicrocontroller and electronics enclosed in a sturdy weatherproof case with LCD display. Operates continuously fo several hours on a single 9 volt alkaline battery.

Packaging: Unit is weather and dust resistant and comes in a rugge case for transportation and shipping.
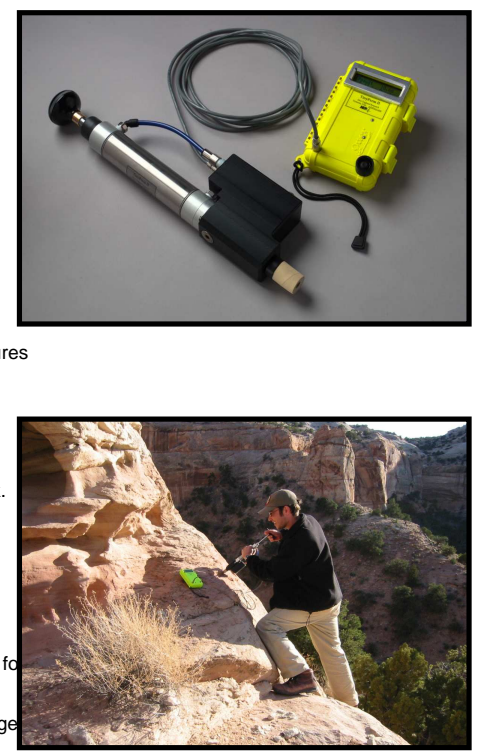

Price: US \$x,xxx

Delivery:45 days

Price and specifications are subject to change.

$\begin{array}{lr}\text { New England Research, Inc. } & \text { http://www.ner.com } \\ 331 \text { Olcott Drive, Suite L1 } & \text { email: info@ner.com } \\ \text { White River Junction, Vermont 05001-9263 } & \text { phone: 802-296-2401 } \\ \text { USA } & \text { fax: 802-296-8333 }\end{array}$




\section{Principal Project Personnel}

\section{Stephen R. Brown}

A. Role in the project: Principal investigator.

\section{B. Curriculum Vita:}

Dr. Stephen Brown is a Principal Scientist at New England Research (1997-present). He was formerly a Senior Scientist at Applied Research Associates (1996-1997), a Member of the Technical Staff at Sandia National Laboratories (1987-1996), a Member of the Professional Staff at Schlumberger-Doll Research (1985-1987), and a post-doctoral research associate at Los Alamos National Laboratory (1984-1985). He received his Ph.D. in geophysics from Columbia University in 1984. His primary research interests are the mechanical, fluid transport, acoustic, and electrical properties of fractured and otherwise heterogeneous rock and the application of geophysical methods to energy and environmental problems. His work combines experimental geophysics in the laboratory, computer modeling, theoretical work, and field work. He has published work on a broad range of topics in geology and geophysics including 24 peer-reviewed research articles in technical journals. He received the 1989 USNCRM/National Academy of Sciences Award for outstanding basic research in geomechanics. His former professional involvements include being a member of the NAS/NRC Committee on Fracture Characterization and Fluid Flow and a member of the board of directors of the American Rock Mechanics Association. He is currently an Adjunct Associate Professor of Geology and Geophysics at the University of Utah, an Adjunct Assistant Professor of Earth Sciences at Dartmouth College, a member of the American Geophysical Union, and a member of the American Institute of Professional Geologists (CPG-10234).

\section{Relevant Publications not emanating from this project:}

Brown, S.R., Fundamental study of the closure property of joints, Ph.D. thesis, Columbia University, 1984.

Brown, S.R., and C.H. Scholz, The closure of random elastic surfaces in contact, J. Geophys. Res., 90, 5531-5545, 1985.

Brown, S.R., and C.H. Scholz, Broad bandwidth study of the topography of natural rock surfaces, $J$. Geophys. Res., 90, 12,575-12,582, 1985.

Brown, S.R., and C.H. Scholz, The closure of rock joints, J. Geophys. Res., 91, 4939-4948, 1986.

Brown, S.R., Fluid flow through rock joints: The effect of surface roughness, J. Geophys. Res., 92, 1337-1347, 1987.

Brown, S.R., A note on the description of surface roughness using fractal dimension, Geophys. Res. Lett., 14, 1095-1098, 1987.

Brown, S.R., Transport of fluid and electric current through a single fracture, J. Geophys. Res., 94, 9429-9438, 1989.

Thompson, M.E., and S.R. Brown, The effect of anisotropic surface roughness on flow and transport in fractures, J. Geophys. Res., 96, 21,923-21,932, 1991.

Olsson, W.A., and S.R. Brown, Hydromechanical response of a fracture undergoing compression and shear, Int. J. Rock Mech. Min. Sci. \& Geomech. Abstr., 30, 845-851, 1993. 
Brown, S.R., Simple mathematical model of a rough fracture, J. Geophys. Res, 100, 5941-5952, 1995.

Yang, G., S.R. Brown, L.R. Myer, and N.G.W. Cook, Microscopic analysis of macroscopic transport properties of single natural fractures using graph theory algorithms, Geophys. Res. Lett., 22, 14291432, 1995.

Brown, S.R., H.W. Stockman, and S.J. Reeves, Applicability of the Reynolds equation for modeling fluid flow between rough surfaces, Geophys. Res. Lett., 22, 2537-2540, 1995.

Brown, S.R., and R.L. Bruhn, Formation of voids and veins during faulting, J. Struct. Geol, 18, 657-671, 1996.

NAS/NRC Committee on Fracture Characterization and Fluid Flow (S.R. Brown, contributing author), Rock Fractures and Fluid Flow: Contemporary Understanding and Applications, National Academy Press, Washington, D.C., 551 pp., 1996.

Brown, S.R., and R.W. Haupt, Study of electrokinetic effects to quantify groundwater flow, Sandia National Laboratories Technical Report no. SAND94-2607, 1997.

Walsh, J.B., S.R. Brown, and W.B. Durham, Effective media theory with spatial correlation for flow in a fracture, J. Geophys. Res., 102, 22,587-22,594, 1997.

Brown, S.R., and R.L. Bruhn, Fluid permeability of deformable fracture networks, J. Geophys. Res., 103, 2489-2500, 1998.

Brown, S., A. Caprihan, and R. Hardy, Experimental observation of fluid flow channels in a single fracture, J. Geophys. Res., 103, 5125-5132, 1998. 


\section{References}

Abelin, H., L. Birgersson, L. Moreno, H. Widen, T. Agren, and I. Neretnieks, A large-scale flow and tracer experiment in granite 2: Results and interpretation, Water Resour. Res., 27, 3119-3135, 1991.

Boullier, A-M., and F. Robert, Paleoseismic events recorded in Archean gold-quartz vein networks, Val d'Or, Abitibi, Quebec, Canada. J. Struct. Geol., 14, 161-179, 1992.

Bourke, P.J., Channeling of flow through fractures in rock, in Proc. of GEOVAL-87, Swedish Nuclear Power Inspectorate, Stockholm, Sweden, 1987.

Brace, W. F., Permeability of crystalline and argillaceous rocks, Int. J. Rock Mech. Min. Sci. Geomech. Abstr., 17, 241-251, 1980.

Brown, S.R., Transport of fluid and electric current through a single fracture, J. Geophys. Res., 94, 94299428, 1989.

Brown, S.R., Simple mathematical model of a rough fracture, J. Geophys. Res., 100, 5941-5952, 1995.

Brown, S.R., Fluid flow through rock joints: The effect of surface roughness, J. Geophys. Res., 92, 13371347, 1987.

Brown, S.R. and R.L. Bruhn, Formation of voids and veins during faulting, J. Struct. Geol, 18, 657-671, 1996.

Brown, S.R., A. Caprihan, and R.D. Hardy, Experimental observation of fluid flow channels in a single fracture, J. Geophys. Res., 103, 5125-5132, 1998.

Brown, S.R., and C.H. Scholz, Broad bandwidth study of the topography of natural rock surfaces. J. Geophys. Res., 90, 12575-12582, 1985.

Brown, S.R., H.W. Stockman, and S.J. Reeves, Applicability of the Reynolds equation for modeling fluid flow between rough surfaces, Geophys. Res. Lett., 22, 2537-2540, 1995.

Burns, K.L., Hot Dry Rock Research, A Compendium of Publications, October 1989 - September 1990, Los Alamos National Laboratory, Hot Dry Rock Project, 1990.

Cacas, M.C., E. Ledoux, G. de Marsily, A. Barbreau, P. Calmels, B. Gaillard, and R. Margritta, Modeling fracture flow with a stochastic discrete fracture network: Calibration and validation 1 . The flow model, Water Resour. Res., 26, 479-489, 1990.

Callaghan, P.T., Principles of Nuclear Magnetic Resonance Microscopy, Clarendon Press, Oxford, 1991.

Caprihan, A., and E. Fukushima, Flow measurements by NMR, Physics Reports (Review Section of Physics Letters), 198, 195- 235, 1990.

Committee on Fracture Characterization and Fluid Flow, National Research Council, Rock Fractures and Fluid Flow: Contemporary Understanding and Applications, National Academy Press, Washington, D.C., 1996.

Detwiler, R. L., R. J. Glass, and W. L. Bourcier, Experimental observations of fracture dissolution: The role of Peclet number on evolving aperture variability, Geophys. Res. Lett. 30(12), 1648-1651, 2003.

Eckel, E., Geology and ore deposits of the La Plata District, CO. U.S. Geol. Surv. Prof. Paper, 219, 1949. 
Endo, H.K., J.C.S. Long, C.R. Wilson, and P.A. Witherspoon, a model for investigating mechanical transport in fracture networks, Water Resour. Res., 20, 1390-1400, 1984.

Fouquet, Y., R. A. Zierenberg, R. A. Miller, Chapter 3 - Middle Valley: Bent Hill Area (Site 1035), Proc. Ocean Drilling Program, Initial Reports, 169, 35-152, 1998.

Gangi, A. F., Variation of whole and fractured porous rock permeability with confining pressure, Int. J. Rock Mech. Min. Sci. Geomech. Abstr., 15, 249-257, 1978.

Guilbert, G.K., and J.M. Park, The Geology of Ore Deposits. W.H. Freeman, New York, 1986.

Harpalani, S., Effect of leaching on microstructure and flow in an in situ mining operation, Mineral Resources Eng., 9, 193-204, 2000.

Huang, C., and D.D. Evans, A three-dimensional computer model to simulate fluid flow and contaminant transport through a rock fracture system, Rep. NUREG/CR-4042, University of Arizona, Tucson, 1985.

Hull, L.C., and K.N. Koslow, Streamline routing through fracture junctions, Water Resour. Res., 22, 17311734, 1986.

Hull, L.C., J.D. Miller, and T.M. Clemo, Laboratory simulation studies of solute transport in fracture networks, Water Resour. Res., 23, 1505-1513, 1987.

Iwai, K., Fundamental studies of fluid flow through a single fracture, Ph.D. thesis, Univ. of Calif., Berkeley, 1976.

Knopf, A., The Mother Lode system of California. U.S. Geol. Surv. Prof. Paper, 157, 103 pp, 1929.

Kranz, R. L., A. D. Frankel, T. Engelder, and C. H. Scholz, The permeability of whole and jointed Barre granite, Int. J. Rock Mech. Min. Sci. Geomech. Abstr., 16, 225-234, 1979.

Nelson, R.A., Geologic Analysis of Naturally Fractured Reservoirs, Gulf Publishing, Houtson, Texas, 320 pp., 1985.

Newhouse, W.H., Openings due to movement along a curved or irregular fault plane. Economic Geology, $35,445-464,1940$.

Olsson, W. A., and S. R. Brown, Hydromechanical response of a fracture undergoing compression and shear, Int. J. Rock Mech. Min. Sci. Geomech. Abstr., 30, 845-851, 1993.

Parney, R., and L. Smith, fluid velocity and path length in fractured media, Geophys. Res. Lett., 22, 14371440, 1995.

Peters, S. G., Formation of oreshoots in mesothermal gold-quartz vein deposits: Examples from Queensland, Australia. Ore Geology Reviews, 8, 277-301, 1993.

Pyrak-Nolte, L. J., N. G. W. Cook, and D. D. Nolte, Fluid percolation through single fractures, Geophys. Res. Lett., 15, 1247-1250, 1988.

Pyrak-Nolte, L. J., Myer, L. R., Cook, N. G. W., and P. A. Witherspoon, Hydraulic and mechanical properties of natural fractures in low permeability rock, in Proceedings of the Sixth International Congress on Rock Mechanics, edited by G. Herget and S. Vongpaisal, pp. 225-231, A. A. Balkema, Rotterdam, Netherlands, 1987. 
Ray, R.G., Geology and ore deposits of the Willow Creek Mining District, Alaska. U.S. Geol. Surv. Bull., 1004, 1954.

Robinson, J.W., and J.E. Gale, A laboratory and numerical investigation of solute transport in discontinuous fracture systems, Groundwater, 28, 25-36, 1990.

Sanderson, D. J., S. Roberts, and P. Gumiel, A Fractal relationship between vein thickness and gold grade in drill core from La Codosera, Spain. Economic Geology, 89, 168-173, 1994.

Sanderson, D. J., and X. Zhang, Critical stress localization of flow associated with deformation of wellfractured rock masses, with implications for mineral deposits, Special Publication of Geological Society London 155, 69-81, 1999.

Schwartz, F.W., L. Smith, and A.S. Crowe, A stochastic analysis of macroscopic dispersion in fractured media, Water Resour. Res., 20, 1253-1265, 1983.

Seymour J.D., and P.T. Callaghan, Generalized Approach to NMR Analysis of Flow and Dispersion in Porous Media, AIChE Journal, 43, 2096-2111, 1997.

Stesky, R. M., Electrical conductivity of brine-saturated fractured rock, Geophysics, 51, 1585-1593, 1986.

Stockman, H.W., A 3D Lattice Boltzmann Code for Modeling Flow and Multi-Component Dispersion, Sandia Laboratories Technical Report, SAND99-0162, February, 1999.

Stockman H. W., J. P. Johnson, and S. R. Brown, Mixing at fracture intersections: influence of channel geometry and the Reynolds and Peclet numbers, Geophys. Res. Lett. 28(22), 4299-4302, 2001.

Stoll, W.C., Relations of structure to deposition at the Independence Mine, Alaska. U.S. Geol. Surv. Bull., 933-C, 201-217, 1940.

Thompson, M.E., and Brown, S.R., The effect of anisotropic surface roughness on flow and transport in fractures. J. Geophys. Res., 96, 21,923-21,932, 1991.

Travis, B.J., TRAC3D: A model of flow and transport in porous fractured media, LA-9667-MS, Los Alamos National Lab, Los Alamos, NM. 1984.

Tsang, Y. W., and P. A. Witherspoon, Hydromechanical behavior of a deformable rock fracture subject to normal stress, J. Geophys. Res., 86, 9287-9298, 1981.

Tsang, Y.W., and C.F. Tsang, Channel model of flow through fractured media, Water Resources Res., 23, 467-479, 1987.

Tsang, Y.W., C.F. Tsang, I. Neretnieks, and L. Moreno, Flow and tracer transport in fractured media: A variable aperture channel model and its properties, Water Resources Res., 23, 467-479, 1988.

U.S. Geological Survey Red-book Conference, Extended Abstracts, The Mechanical Involvement of Fluids in Faulting, Tenaya Lodge, Fish Camp, California, June 6-10, 1993.

Walsh, J. B., Effect of pore pressure and confining pressure on fracture permeability, Int. J. Rock Mech. Min. Sci. Geomech. Abstr., 18, 429-435, 1981.

Walsh, J. B., S.R. Brown, and W.B. Durham, Effective media theory with spatial correlation for flow in a fracture, J. Geophys. Res., 102, 22,587-22,594, 1997. 
Wilson, C.R., and P.A. Witherspoon, Flow interference effects at fracture intersections, Water Resour. Res., 12, 102-104, 1976.

$\mathrm{Xu}, \mathrm{T}$., and K. Preuss, On flow and mineral alteration in fractured caprock of magmatic hydrothermal systems, J. Geophys. Res., 106, 2121-2138, 2001.

Zimmerman, R. W., D-W. Chen, and N. G. W. Cook, The effect of contact area on the permeability of fractures, J. Hydrol., 139, 79-96, 1992. 